\title{
Outsourcing versus technology transfer: Hotelling meets Stackelberg*
}

\author{
Andrea Pierce ${ }^{\dagger}$ DEBAPRiYA SEN ${ }^{\ddagger}$
}

September 29, 2009

\begin{abstract}
This paper considers a Hotelling duopoly with two firms $A$ and $B$ in the final good market. Both $A$ and $B$ can produce the required intermediate good, firm $B$ having a lower cost due to a superior technology. We compare two contracts: outsourcing ( $A$ orders the intermediate good from $B$ ) and technology transfer $(B$ transfers its technology to $A$ ). First we show that an outsourcing order acts as a credible commitment on part of $A$ to maintain a certain market share in the final good market. This generates an indirect Stackelberg leadership effect, which is absent in a technology transfer contract. We show that compared to the situation of no contracts, there are always Pareto improving outsourcing contracts but no Pareto improving technology transfer contracts. Finally, it is shown that whenever both firms prefer one of the two contracts, all consumers prefer the other contract.
\end{abstract}

Keywords: Outsourcing, Technology transfer, Hotelling duopoly, Stackelberg effect, Pareto improving contracts

JEL Classification: D43, L11, L13

*For helpful comments, we thank the participants at the 2009 CEA annual conference in Toronto.

${ }^{\dagger}$ Department of Economics, Ryerson University, 350 Victoria Street, Toronto, Ontario M5B 2K3, Canada. Email: akpierce@ryerson.ca

${ }_{\ddagger}^{\ddagger}$ Department of Economics, Ryerson University, 350 Victoria Street, Toronto, Ontario M5B 2K3, Canada. Email: dsen@arts.ryerson.ca 


\section{Introduction}

In this era of globalization, it has become increasingly common for firms to outsource their required inputs rather than produce them in-house. While many factors influence a firm's decision to outsource (see, e.g., Jarillo, 1993; Domberger, 1998 for a discussion of these factors), it can be argued that outsourcing is primarily driven by cost considerations. A firm will choose to outsource if the input supplier can offer a price that is lower than the firm's in-house cost. This will be the case if the supplier has a cost advantage in one or more factors of production. Such advantages can be interpreted broadly as the supplier having a superior production technology. For example, the supplier may be able to hire skilled labour at a relatively low wage or it may possess advanced machineries. It is therefore plausible that transfer of technology across firms could be a viable alternative to outsourcing. In fact, like outsourcing, technology transfers have grown substantially in recent years (see, e.g., Mendi, 2005; Vishwasrao, 2007). ${ }^{1}$ As outsourcing and technology transfer generally serve the same purpose (i.e., enabling one firm to use the cost-efficient production process of another firm), a natural question is, what would make firms choose one of these contracts over another? There could be different possible reasons to prefer outsourcing. For instance, the superior technology may be labour intensive and difficult to transfer due to imperfect mobility of labour. Additionally, transfer of technology may involve large initial investments that firms may want to avoid. On the other hand, under technology transfer, a firm produces its inputs in-house using the superior technology which gives it complete control over its production. This will be preferred by a firm if it has a higher quality standard or if it wants to avoid the risk of relying on another firm for its inputs.

Apart from the reasons given above, strategic considerations play an important role in determining the nature of input production decisions of a firm. The strategic motive will be particularly dominant when the input-seeking firm competes with the supplier in the final good market. This paper aims to shed light on these strategic aspects in a model of price competition. Specifically, we consider a Hotelling duopoly with two firms, $A$ and $B$, who are located at the end points of the unit interval in the final good market $\varphi$. Consumers are uniformly distributed in this interval and have to incur transportation costs for traveling to the end points. Any consumer buys one unit of $\varphi$ from either $A$ or $B$, or chooses a specific outside option. We carry out our analysis under two alternative scenarios: the case of the covered market where all consumers buy from either $A$ or $B$, and the case of the uncovered market where some consumers do not buy from either of these firms and instead choose the outside option. We employ the Hotelling model, because it is versatile in that it has multiple interpretations that apply to diverse economic situations as illustrated below. ${ }^{2}$

When the outside option is sufficiently unappealing, it is not optimal for consumers to choose that option. This can correspond to a situation where the good produced by firms $A$ and $B$ is an essential good for the consumers. Accordingly, any consumer will buy the

\footnotetext{
${ }^{1}$ For example, commenting on international technology transfer by U.S. firms, Vishwasrao (2007, p. 742-743) points out: “...in 2002, U.S. companies collected $\$ 29,023$ million in royalties and fees from their foreign subsidiaries and an additional $\$ 12,075$ million in royalties and fees from unaffiliated firms in foreign countries. These receipts have roughly doubled over the last decade. For 2002, the royalty and fee receipts of US multinational companies represent almost $20 \%$ of the foreign direct investment income for the US."

${ }^{2}$ For a more detailed discussion on different interpretations of the Hotelling model, see Anderson et al. (1992) and Gabszewicz and Thisse (1992).
} 
good from either $A$ or $B$ and the market will be covered. For example, the unit interval can represent an underdeveloped village and the end points can correspond to two neighboring towns where firms $A$ and $B$ are located. The good could be an essential facility such as a railway station or a post office. Thus, each town has a station or an office that is served by one of the two firms. For this example the location in the Hotelling model represents physical location and for any consumer in the village, the transportation cost is literally the cost of traveling to one of the towns to use the essential facility.

On the other hand, when the outside option is sufficiently appealing, it can correspond to an existing good while good $\varphi$, produced by firms $A$ and $B$, can be viewed as an updated and differentiated version of the existing good. For example, the existing good could be a household cleaner which contains harmful toxins, while good $\varphi$ could be an environmentally friendly cleaner. Firms $A$ and $B$ specialize in producing two different types of the non-toxic cleaner. In this example, the location represents a consumer's taste. Consumers who are relatively close to one of the end points prefer the product characteristics offered by the firm located at that point and buys from that firm. However, consumers who are not very close to either of the end points do not have a strong preference for the characteristics of the upgraded product $\varphi$ and continue to use the existing product. These consumers do not buy from either $A$ or $B$ and as a result, the market is not covered.

Apart from the versatility of the Hotelling model, the duopoly structure of this paper encompasses various possibilities. For example, both firms $A$ and $B$ could be local firms competing in a domestic market. Alternatively, one of them could be a foreign firm, or both could be multinationals competing in a third country.

We consider a production process where an intermediate good $\eta$ is required to manufacture $\varphi$. Each firm can convert one unit of good $\eta$ into one unit of good $\varphi$ at zero cost. Both $A$ and $B$ can produce $\eta$, but firm $B$ has a lower cost due to a superior technology. We study two contracts that naturally arise in this situation: outsourcing (firm $A$ orders $\eta$ from firm $B$ ) and technology transfer (firm $B$ transfers its technology to firm $A$ ). Unit-based pricing policies are considered for both cases, where the unit price is determined through negotiations between firms $A$ and $B$. Under an outsourcing contract, firm $A$ can place any order with firm $B$ at the agreed upon price. Under a technology transfer contract, firm $A$ uses the superior technology of firm $B$ by paying a price for each unit of production, i.e., the technology transfer contract is based on a unit royalty. ${ }^{3}$ To compare these two contracts, we denote $\omega$ to be the effective unit cost of firm $A$ in any contract. Fixing $\omega$, we compare the contracts for both uncovered and covered markets.

In the uncovered scenario, each firm has a local monopoly over a distinct segment of the market $\varphi$. Consequently, firm B's supply of $\eta$ to firm $A$ does not affect $B$ 's profit in the market $\varphi$. Due to this reason, outsourcing and technology transfer lead to the same outcome. For any $\omega$, both firms and all consumers are indifferent between these two contracts. Moreover, compared to the situation of no contracts, each of these contracts is weakly Pareto improving: each contract makes both firms better off, some consumers better off and no consumers worse off.

Under covered markets, the interaction between firms $A$ and $B$ in the market $\varphi$ is strategic

\footnotetext{
${ }^{3}$ We consider unit pricing policies for outsourcing and unit royalty policies for technology transfer because they are most frequently observed in practice. See Robinson and Kalakota (2004) and Vagadia (2007) for evidence on outsourcing and Mendi (2005), Nagaoka (2005) and Vishwasrao (2007) for technology transfer.
} 
in that the price set by a firm affects the market share and profit of its rival. Due to this strategic aspect, outsourcing and technology transfer contracts generate different incentives for any firm. We show that this difference alters the strategic interaction between firms and has important implications on prices.

Specifically we show that compared to the case of no contracts, prices never rise under outsourcing while this is not necessarily the case under technology transfer. There are always weakly Pareto improving outsourcing contracts that make both firms better off and no consumers worse off. Moreover if the cost difference of firms is relatively large, there are strictly Pareto improving outsourcing contracts that make both firms as well as all consumers better off. On the other hand, there are no Pareto improving technology transfer contracts: whenever both firms prefer technology transfer over no contracts, there are always some consumers who are worse off. Finally we show that due to the difference in strategic interaction between these two contracts, the incentives of firms and the interest of consumers move in the opposite direction. For any $\omega$, whenever both firms prefer one of these two contracts, all consumers prefer the other.

When firm $A$ outsources $\eta$ to firm $B$, Hotelling meets Stackelberg. The volume of the outsourcing order plays an interesting role of information transmission because it credibly informs $B$ that $A$ is committed to maintain a specific market share in the market $\varphi$. This market share can be viewed as the Stackelberg leader market share. ${ }^{4}$ When the unit price $\omega$ is small, the Stackelberg market share is larger than $A$ 's market share under no contracts. This large share is sustained in equilibrium by a lower price of firm $A$. Given that $A$ 's commitment of Stackelberg market share is credible, $B$ 's equilibrium price is also set lower and prices of both firms fall. For firm $B$, this results in lower profit in the market $\varphi$ compared to the case of no contracts. So $B$ would accept such an outsourcing contract only if it can obtain a large supplier profit from $\eta$ to compensate for its losses in the market $\varphi$. This will be the case when firm $B$ is sufficiently more efficient compared to firm $A$. Consequently, when the cost difference of two firms is sufficiently large, there are strictly Pareto improving outsourcing contracts, i.e., both firms prefer outsourcing over no contracts and both set a lower price for $\varphi$ that makes all consumers better off.

Under technology transfer, firm $A$ acquires $B$ 's superior technology and produces $\eta$ itself using this technology. As a result, firm $B$ knows the quantity of $\eta$ produced by $A$ only when it receives its payments for technology transfer. As these payments are received after profits are realized in the final good market, the informational aspect in outsourcing is completely absent under technology transfer. Moreover since firm $B$ 's payments from the transfer depends on the demand of $A$, it has an incentive to ensure that $A$ 's demand is not too small. This creates a distortion that raises the effective cost of $B$ which in turn adversely affects prices in the market $\varphi$. Due to this distortion, technology transfer contracts that are preferred by both firms necessarily make some consumers worse off. Consequently, unlike the case of outsourcing, there are no Pareto improving technology transfer contracts in relation to the situation of no contracts. The difference between outsourcing and technology transfer is driven by two factors: first, the information transmission and the subsequent Stackelberg leadership effect that leads to lower prices under outsourcing is absent under technology

\footnotetext{
${ }^{4}$ When firms $A$ and $B$ compete in quantities as Cournot duopolists, $A$ 's outsourcing order corresponds to the Stackelberg leader output (see Baake et al., 1995; Chen et al., 2009). The leadership effect is indirect under price competition: following the outsourcing order by $A$, equilibrium prices are formed in a way so that $A$ 's market share exactly equals the quantity of $\eta$ it has ordered from $B$.
} 
transfer, and second, outsourcing orders are obtained upfront before firms set their prices, so outsourcing has no distortive effect.

The existing literature has studied horizontal outsourcing (i.e., a firm outsources to its rival) under different models of price competition such as a Hotelling duopoly (Shy and Stenbacka, 2003), ${ }^{5}$ a duopoly with differentiated products (Chen et al., 2004) and a Bertrand duopoly (Arya et al., 2007). It is concluded in these papers that horizontal outsourcing is inefficient and leads to higher prices in the final good market. To a certain extent, we obtain similar implications under technology transfer where we show that there are no Pareto improving contracts. However, under outsourcing, we show that prices never rise and there are always Pareto improving contracts. Thus, our result on outsourcing differs with the conclusion of the existing literature.

This difference arises because the existing literature generally treats outsourcing and technology transfer equivalently. Specifically, it overlooks the informational aspect of outsourcing which is the main focus of this paper. The information transmission in outsourcing is driven by our presumed sequence of events. In our model, firm $A$ places its outsourcing order of $\eta$ first and then prices are set in the market $\varphi$. The papers mentioned above implicitly assumes an alternative sequence where the input-seeking firm places its outsourcing order with its rival after firms set their prices in the final good market. Under this sequence, outsourcing does not transmit any information to the supplier firm prior to price competition. Since outsourcing order is not received upfront, to obtain higher profit from outsourcing the supplier has to ensure that the input-seeking firm's demand is not too small. As a result, the distortive effect of technology transfer is present in outsourcing which explains why outsourcing contracts are inefficient under the sequence assumed in the literature.

It can be argued that our presumed sequence is more realistic. An outsourcing order takes time to process for reasons such as logistics. Additionally, compared to in-house production, an outsourcing order may have to pass through more stages of inspection. These factors will be particularly dominant if the supplier is a foreign firm, which has been a frequently observed occurrence in recent years. Therefore, if a firm places its outsourcing order after receiving its demand, it may not be able to meet its demand on time. For this reason, it is natural to assume that firms negotiate and sign an outsourcing contract well in advance before the final goods market meets. This approach is also consistent with the literature of outsourcing under quantity competition. In these models, the input-seeking firm chooses its outsourcing order first and then firms choose quantities in the final good market. The Stackelberg leadership effect is direct under quantity competition: by placing an outsourcing order with a rival firm in a Cournot duopoly, the input-seeking firm can establish itself as the Stackelberg leader (see Baake et al., 1995; Chen et al. 2009).

This paper is also related to the literature of capacity commitments in duopolies. When firm $A$ places an outsourcing order with firm $B$, the volume of the order can be viewed as a capacity that $A$ builds prior to price competition. If the demand of $A$ in the market $\varphi$ does not exceed its capacity, it can meet its demand at zero marginal cost. However, if the demand exceeds the capacity, $A$ meets the additional demand by producing $\eta$ in-house that raises its marginal cost. We show that in equilibrium, firm $A$ 's demand exactly equals its capacity. This implies that $A$ does not produce $\eta$ in-house and there is no unutilized capacity. Thus,

${ }^{5}$ The primary focus of Shy and Stenbacka (2003) is vertical outsourcing (i.e. firms outsource to an outside supplier), although they consider horizontal outsourcing as well. 
an outsourcing contract in this paper is a natural quantity precommitment in the spirit of Kreps and Scheinkman (1983). They have shown that if firms in a Bertrand duopoly can make such commitments by building prior capacities, then prices rise to the Cournot level. This paper presents an interesting contrast by showing that when the precommitment is made by an input-seeking firm through an outsourcing order, prices either fall or stay the same.

The paper is organized as follows. We present the model in Section 2. Three contractual settings (no contracts, outsourcing and technology transfer) are studied in Section 3. We conclude in Section 4. Most proofs are relegated to the Appendix.

\section{The model}

The final good market: The market for the final good $\varphi$ is a linear city Hotelling duopoly with two firms $A$ and $B$. Firm $A$ is located at point 0 and firm $B$ at point 1 of the unit interval $[0,1]$. Firms compete in prices.

Consumers are uniformly distributed in $[0,1]$. Any consumer buys either one unit of good $\varphi$ or chooses a specific outside option. Consumers receive utility $V>0$ from good $\varphi$. The outside option gives the utility $V_{0}<V$. Therefore, a consumer who does not purchase good $\varphi$ from either $A$ or $B$ obtains utility $V_{0}$.

The unit cost of transportation is $\tau>0$. For a consumer at location $x \in[0,1]$, the transportation cost to travel to firm $A$ is $\tau x$, while the cost to travel to firm $B$ is $\tau(1-x)$. A consumer who buys one unit of good $\varphi$ from either $A$ or $B$ gets utility $V$, pays the price and incurs the cost of transportation.

Let $p_{A}, p_{B} \geq 0$ be the prices set by firms $A, B$ and denote $p \equiv\left(p_{A}, p_{B}\right)$. Given any $p$, a consumer has the set of purchasing options $\mathcal{C}=\{A, B, 0\}$ where

$$
A \equiv[\text { buy } \varphi \text { from } A], B \equiv[\text { buy } \varphi \text { from } B] \text { and } 0 \equiv[\text { choose the outside option }]
$$

For $z \in \mathcal{C}$, the net utility of a consumer at location $x \in[0,1]$ is

$$
u_{x}^{p}(z)= \begin{cases}V-p_{A}-\tau x & \text { if } z=A \\ V-p_{B}-\tau(1-x) & \text { if } z=B \\ V_{0} & \text { if } z=0\end{cases}
$$

For any $p$, a consumer at location $x$ chooses $z \in \mathcal{C}$ to maximize $u_{x}^{p}(z)$. We shall consider two alternative scenarios:

(i) The case of the covered market where all consumers will purchase good $\varphi$ from either $A$ or $B$.

(ii) The case of the uncovered market where some consumers will not purchase $\varphi$ from $A$ or $B$ and will choose the outside option.

Whether the market will be covered or not depends on the utility from the outside option $V_{0}$. If $V_{0}$ is sufficiently small, the market will be covered and if $V_{0}$ is sufficiently large, it will be uncovered. We shall carry out our analysis under the following alternative assumptions. 
Covered Assumption (CA) $V_{0}=-\infty$

Uncovered Assumption (UA) $V_{0}>V-\tau / 2$

Under the covered assumption $(\mathrm{CA})$, any consumer receives a large negative utility $V_{0}$ from the outside option. Therefore, it is not optimal for any consumer to choose the outside option. A consumer at location $x$ will choose either $A$ or $B$ by comparing $u_{x}^{p}(A)$ and $u_{x}^{p}(B)$ from (1). Consequently, the market will be covered.

Now suppose the uncovered assumption (UA) holds. Consider the consumer who is located exactly in the middle of the unit interval $[0,1]$, i.e., at $x=1 / 2$. This consumer incurs the same transportation cost $\tau / 2$ for traveling to either $A$ or $B$. When $p_{A}=p_{B}=0$, this consumer obtains the same net utility $V-\tau / 2<V_{0}$ from $A$ or $B$. Thus, even when firms set zero prices, the consumer at $x=1 / 2$ strictly prefers the outside option over both $A$ and $B$. Therefore, for any $p$, this consumer will choose the outside option. Then it follows that for any $p$, there will be always some consumers located in a close neighbourhood of $1 / 2$ who will also prefer the outside option over both $A$ and $B$. That is, there will be always an interval $[a, b](0 \leq a<1 / 2<b \leq 1)$ such that all consumers at location $x \in[a, b]$ will choose the outside option. As a result, the market will be uncovered.

Demand of firms: Let $D_{A}(p)$ and $D_{B}(p)$ be the demand received by firms $A, B$ when they set prices $p_{A}, p_{B}$.

Demand under CA: Under CA, it is optimal for any consumer to choose either $A$ or $B$. It follows from (1) that $u_{x}^{p}(A) \gtreqless u_{x}^{p}(B) \Leftrightarrow x \lesseqgtr \widetilde{x}(p)$ where

$$
\widetilde{x}(p)=\left(p_{B}-p_{A}+\tau\right) / 2 \tau
$$

So a consumer at location $x \in[0,1]$ chooses $A$ if $x \leq \widetilde{x}(p)$ and $B$ if $x>\widetilde{x}(p)$. Observe that if $p_{A} \geq p_{B}+\tau$, then $\widetilde{x}(p) \leq 0$ and all consumers choose $B$. On the other hand, if $p_{B} \geq p_{A}+\tau$, then $\widetilde{x}(p) \geq 1$ and all consumers choose $A$. If $p_{A}<p_{B}+\tau$ and $p_{B}<p_{A}+\tau$, then $0<\widetilde{x}(p)<1$. In that case, consumers at location $x \in[0, \widetilde{x}(p)]$ choose $A$ and $x \in(\widetilde{x}(p), 1]$ choose $B$. Hence we conclude that

$$
\left(D_{A}(p), D_{B}(p)\right)= \begin{cases}(0,1) & \text { if } p_{A} \geq p_{B}+\tau \\ (1,0) & \text { if } p_{B} \geq p_{A}+\tau \\ (\widetilde{x}(p), 1-\widetilde{x}(p)) & \text { if } p_{A}<p_{B}+\tau \text { and } p_{B}<p_{A}+\tau\end{cases}
$$

Demand under UA (two local monopolies): Now suppose UA holds. From (1), $u_{x}^{p}(A) \gtreqless V_{0} \Leftrightarrow x \lesseqgtr \widetilde{x}_{A}\left(p_{A}\right)$ and $u_{x}^{p}(B) \gtreqless V_{0} \Leftrightarrow x \gtreqless \widetilde{x}_{B}\left(p_{B}\right)$ where

$$
\widetilde{x}_{A}\left(p_{A}\right)=\left(V-V_{0}-p_{A}\right) / \tau \text { and } \widetilde{x}_{B}\left(p_{B}\right)=1-\left(V-V_{0}-p_{B}\right) / \tau
$$

If $p_{A} \geq V-V_{0}$, then $\widetilde{x}_{A}\left(p_{A}\right) \leq 0$ and no consumer chooses $A$. On the other hand, if $p_{B} \geq V-V_{0}$, then $\widetilde{x}_{B}\left(p_{B}\right) \geq 1$ and no consumer chooses $B$. If both $p_{A}, p_{B}$ are less than $V-V_{0}$, then $\widetilde{x}_{A}\left(p_{A}\right)>0, \widetilde{x}_{B}\left(p_{B}\right)<1$ and by UA,

$$
\widetilde{x}_{A}\left(p_{A}\right)<1 / 2<\widetilde{x}_{B}\left(p_{B}\right)
$$

We know that under UA, the consumer located at $x=1 / 2$ always prefers the outside option over both $A$ and $B$. Since $u_{x}^{p}(A)$ is decreasing and $u_{x}^{p}(B)$ is increasing in $x$, it follows that all consumers at $x \in[1 / 2,1]$ prefer the outside option over $A$ and all consumers at $x \in[0,1 / 2]$ prefer the outside option over $B$. Using this fact together with (5), we conclude: 
(i) Consumers at $x \in\left[0, \widetilde{x}_{A}\left(p_{A}\right)\right)$ choose $A$, so $D_{A}(p)=\widetilde{x}_{A}\left(p_{A}\right)=\left(V-V_{0}-p_{A}\right) / \tau$

(ii) Consumers at $x \in\left(\widetilde{x}_{B}\left(p_{B}\right), 1\right]$ choose $B$, so $D_{B}(p)=1-\widetilde{x}_{B}\left(p_{B}\right)=\left(V-V_{0}-p_{B}\right) / \tau$

(iii) Consumers at $x \in\left[\widetilde{x}_{A}\left(p_{A}\right), \widetilde{x}_{B}\left(p_{B}\right)\right]$ choose the outside option

Therefore, under UA, the demand received by a firm depends only on its price and does not depend at all on the price of its rival. Accordingly, each firm $i \in\{A, B\}$ becomes a local monopolist. The demand that firm $i$ receives when it sets price $p_{i}$ is the monopoly demand $D_{M}\left(p_{i}\right)$. Hence,

$$
D_{i}(p)=D_{M}\left(p_{i}\right)= \begin{cases}\left(V-V_{0}-p_{i}\right) / \tau & \text { if } p_{i}<V-V_{0} \\ 0 & \text { if } p_{i} \geq V-V_{0}\end{cases}
$$

The intermediate good: An intermediate good $\eta$ is required to produce $\varphi$. Both firms can convert one unit of good $\eta$ into one unit of good $\varphi$ at the same constant marginal cost, which we normalize to zero.

The constant marginal cost of production of good $\eta$ is $\bar{c}>0$ for $A$ and $\underline{c}>0$ for $B$. Firm $B$ has a superior technology for producing $\eta$, so its cost is lower, i.e., $\underline{c}<\bar{c}$. We also assume that the costs are sufficiently small. Specifically, it is assumed that

$$
0<\underline{c}<\bar{c}<\min \left\{V-V_{0}, \tau\right\}
$$

Observe that (7) reduces to

$$
0<\underline{c}<\bar{c}<\tau \text { under } \mathrm{CA} \text { and } 0<\underline{c}<\bar{c}<V-V_{0} \text { under } \mathrm{UA}
$$

The effective unit cost of $\eta$ for a firm will depend on the nature of contracts that $A$ and $B$ have in the intermediate good market. We consider the following possibilities:

(i) No contract between $A$ and $B$

(ii) Outsourcing contract between $A$ and $B$ : $A$ orders $\eta$ from $B$

(iii) Technology transfer from $B$ to $A$ : firm $B$ transfers its superior technology of producing $\eta$ to firm $A$

Before formally describing the three contractual situations above, it will be useful for our analysis to introduce the Hotelling duopoly game $\mathbb{H}\left(c_{A}, c_{B}\right)$.

The Hotelling duopoly game $\mathbb{H}\left(c_{A}, c_{B}\right)$ : This is the standard Hotelling duopoly game played between firms $A$ and $B$ in the final good market $\varphi$, where the constant unit cost of producing the intermediate good $\eta$ is $c_{A} \geq 0$ for firm $A$ and $c_{B} \geq 0$ for firm $B$ and each firm can transform one unit of $\eta$ to one unit of $\varphi$ at zero cost. This game has the following stages.

Stage 1: Firms $A$ and $B$ simultaneously set prices $p_{A}, p_{B} \geq 0$. For any $p \equiv\left(p_{A}, p_{B}\right)$, firm $i$ receives the demand $D_{i}(p)$, which is given by (3) under CA and by (6) under UA. 
Stage 2: Observing $D_{i}(p)$, firms $A$ and $B$ simultaneously choose $q_{A}, q_{B} \geq 0$ where

$$
q_{i}=\text { the quantity of } \eta \text { that firm } i \text { produces in order to fulfill its demand of } \varphi \text {. }
$$

The demand fulfilling constraints are $q_{A} \geq D_{A}(p)$ and $q_{B} \geq D_{B}(p)$. If the cost of producing $\eta$ is positive for firm $i$, optimality requires that it produces $q_{i}=D_{i}(p)$ units of $\eta$ and transforms these $D_{i}(p)$ units to good $\varphi$ to fulfill its demand. If the cost is zero, it is optimal for $i$ to produce any $q_{i} \geq D_{i}(p)$ units of $\eta$ and transform $D_{i}(p)$ units to good $\varphi$ to fulfill its demand. In either case, the payoff (profit) functions of $A$ and $B$ in $\mathbb{H}\left(c_{A}, c_{B}\right)$ are $\Phi_{A}(p)=\left(p_{A}-c_{A}\right) D_{A}(p)$ and $\Phi_{B}(p)=\left(p_{B}-c_{B}\right) D_{B}(p)$.

Lemma 1 characterizes Subgame Perfect Nash Equilibrium (SPNE) of $\mathbb{H}\left(c_{A}, c_{B}\right)$.

Lemma 1 Suppose $c_{A}, c_{B} \in\left[0, \min \left\{V-V_{0}, \tau\right\}\right)$. If either $C A$ or $U A$ holds, then $\mathbb{H}\left(c_{A}, c_{B}\right)$ has a unique SPNE. For $i \in\{A, B\}$, let $p_{i}\left(c_{A}, c_{B}\right), D_{i}\left(c_{A}, c_{B}\right)$ and $\Phi_{i}\left(c_{A}, c_{B}\right)$ be the SPNE price, market share and profit of firm $i$.

(I) If UA holds, then firms $A$ and $B$ become two local monopolists. Firm $i \in\{A, B\}$ sets the monopoly price $\rho_{M}\left(c_{i}\right)$, receives the monopoly demand $\delta_{M}\left(c_{i}\right)$ and obtains the monopoly profit $\psi_{M}\left(c_{i}\right)$, where

$$
\rho_{M}\left(c_{i}\right)=\left(V-V_{0}+c_{i}\right) / 2, \delta_{M}\left(c_{i}\right)=\left(V-V_{0}-c_{i}\right) / 2 \tau \text { and } \psi_{M}\left(c_{i}\right)=\left(V-V_{0}-c_{i}\right)^{2} / 4 \tau
$$

(II) If CA holds, then $p_{A}=\tau+\left(2 c_{A}+c_{B}\right) / 3, p_{B}=\tau+\left(c_{A}+2 c_{B}\right) / 3 ; D_{A}=1 / 2-\left(c_{A}-c_{B}\right) / 6 \tau$, $D_{B}=1 / 2+\left(c_{A}-c_{B}\right) / 6 \tau ; \Phi_{A}=\left(3 \tau-c_{A}+c_{B}\right)^{2} / 18 \tau, \Phi_{B}=\left(3 \tau+c_{A}-c_{B}\right)^{2} / 18 \tau$. The market is covered, i.e., $D_{A}+D_{B}=1$.

Proof (I) Observe from (6) that under UA, firm $i \in\{A, B\}$ chooses its price $p_{i}$ to maximize its local monopolist's profit $\left(p_{i}-c_{i}\right) D_{M}\left(p_{i}\right)$. Since $c_{i}<V-V_{0}$, it follows from (6) that it is optimal for firm $i$ to set the monopoly price $\rho_{M}\left(c_{i}\right)$ given in (9). The expressions of monopoly demand $\delta_{M}\left(c_{i}\right)$ and profit $\psi_{M}\left(c_{i}\right)$ are immediate.

(II) See the Appendix.

\section{Three contractual settings}

\subsection{No contracts between $A$ and $B$}

When there are no contracts between firms $A$ and $B$ in the intermediate good market $\eta$, firm $A$ produces $\eta$ under cost $\bar{c}$, while firm $B$ has cost $\underline{c}$. Accordingly, the Hotelling duopoly game $\mathbb{H}(\bar{c}, \underline{c})$ is played between $A$ and $B$ in the market $\varphi$.

Proposition 1 When there are no contracts between firms $A$ and $B$, the Hotelling duopoly game $\mathbb{H}(\bar{c}, \underline{c})$ is played. This game has a unique $S P N E$. For $i \in\{A, B\}$, let $p_{i}^{0}, D_{i}^{0}$ and $\Phi_{i}^{0}$ be the SPNE price, demand and profit of firm $i$.

(I) If UA holds, then $p_{A}^{0}=\rho_{M}(\bar{c}), p_{B}^{0}=\rho_{M}(\underline{c}) ; D_{A}^{0}=\delta_{M}(\bar{c}), D_{B}^{0}=\delta_{M}(\underline{c}) ; \Phi_{A}^{0}=\psi_{M}(\bar{c})$, $\Phi_{B}^{0}=\psi_{M}(\underline{c})$. Firms $A$ and $B$ become local monopolists and the market is uncovered, i.e., $D_{A}^{0}+D_{B}^{0}=\delta_{M}(\bar{c})+\delta_{M}(\underline{c})<1$. 
(II) Denote $\underline{\theta} \equiv(2 \underline{c}+\bar{c}) / 3$ and $\bar{\theta} \equiv(\underline{c}+2 \bar{c}) / 3$. If $C A$ holds, then $p_{A}^{0}=\tau+\bar{\theta}, p_{B}^{0}=\tau+\underline{\theta}$; $D_{A}^{0}=1 / 2-(\bar{c}-\underline{c}) / 6 \tau, D_{B}^{0}=1 / 2+(\bar{c}-\underline{c}) / 6 \tau ; \Phi_{A}^{0}=(3 \tau-\bar{c}+\underline{c})^{2} / 18 \tau ; \Phi_{B}^{0}=(3 \tau+\underline{c}-\bar{c})^{2} / 18 \tau$. The market is covered, i.e., $D_{A}^{0}+D_{B}^{0}=1$.

Proof Follows from Lemma 1 by taking $c_{A}=\bar{c}$ and $c_{B}=\underline{c}$.

\subsection{Outsourcing contract between $A$ and $B$}

When there is an outsourcing contract between $A$ and $B$, firm $A$ has two options of acquiring the intermediate good $\eta$ : (i) it can order $\eta$ from firm $B$ or (ii) it can produce this good itself at cost $\bar{c}$. We do not impose any exclusivity restriction on outsourcing contracts. That is, firm $A$ can order $\eta$ from firm $B$ as well as produce it in-house. Firm $B$ produces its required $\eta$ entirely by itself at cost $\underline{c}$.

We consider linear unit pricing contracts: firm $B$ charges a constant price for each unit of $\eta$ that it supplies to firm $A$. The unit price is determined through negotiations between firms $A$ and $B$. Under outsourcing contracts, the strategic interaction between $A$ and $B$ is described as follows.

Negotiation stage: In the beginning, firms $A$ and $B$ negotiate on the unit price $\omega$ at which $B$ can supply $\eta$ to $A$. As firm $A$ can produce $\eta$ itself at unit cost $\bar{c}$, an outsourcing contract can lower its cost of production only if $\omega<\bar{c}$. On the other hand, as firm $B$ 's unit cost of $\eta$ is $\underline{c}$, it obtains a positive profit as a supplier only if $\omega>\underline{c}$. For this reason, we restrict $\omega \in(\underline{c}, \bar{c})$.

If firms do not agree on a price, firm $A$ produces the required $\eta$ entirely by itself at cost $\bar{c}$ and the game $\mathbb{H}(\bar{c}, \underline{c})$ is played in the final good market. If firms agree on a price $\omega \in(\underline{c}, \bar{c})$, the game $\Gamma^{S}(\omega)$ is played between $A$ and $B$.

The game $\Gamma^{S}(\omega)$ : It is an extensive form game that has the following stages.

Stage I: Firm $A$ chooses the amount $K \in[0,1]$ of $\eta$ to $\operatorname{order}^{6}$ from firm $B$. Firm $A$ receives $K$ units of $\eta$ by paying $\omega K$ to firm $B$ and $B$ obtains the supplier profit $(\omega-\underline{c}) K$.

Stage II: Firms $A$ and $B$ play the Hotelling duopoly game $\mathbb{H}^{K}(\bar{c}, \underline{c})$. This game has the following stages.

Stage 1: Firms $A$ and $B$ simultaneously announce prices $p_{A}, p_{B}$ for the final good market $\varphi$. For any $p \equiv\left(p_{A}, p_{B}\right)$, the demand received by firm $i \in\{A, B\}$ is $D_{i}(p)$, given by (3) under $\mathrm{CA}$ and (6) under UA.

Stage 2: Observing $D_{i}(p)$, firms $A$ and $B$ simultaneously choose $q_{A}, q_{B} \geq 0$ where

$$
q_{i}=\text { the quantity of } \eta \text { that firm } i \text { produces in order to fulfill its demand of } \varphi
$$

As $A$ already has $K$ units of $\eta$ from stage 1, its demand fulfilling constraint is

$$
K+q_{A} \geq D_{A}(p)
$$

\footnotetext{
${ }^{6}$ Since the maximum demand that a firm can have is 1 , there is no loss of generality in restricting $K \leq 1$. In our model firms $A$ and $B$ negotiate on the price $\omega$ and then $A$ chooses the outsourcing order $K$. Alternatively, one can allow $A$ and $B$ to negotiate on both $\omega$ and $K$. Our qualitative conclusions remain unaltered under this alternative.
} 
As firm $B$ produces $\eta$ entirely by itself, the corresponding constraint for $B$ is

$$
q_{B} \geq D_{B}(p)
$$

Each firm fulfills its demand, profits are realized and the game terminates.

Since the unit cost of producing $\eta$ is positive for each firm, by (11), optimality requires that firm $B$ produces $q_{B}=D_{B}(p)$ units of $\eta$ and transforms $D_{B}(p)$ units to good $\varphi$ to fulfill its demand. Regarding firm $A$, by $(10)$, if $D_{A}(p) \leq K$ (i.e. its demand does not exceed the amount of $\eta$ it has ordered from $B$ ), then it is optimal for $A$ to choose $q_{A}=0$ (i.e. it does not produce $\eta$ in-house) and transform $D_{A}(p)$ units of $\eta$ to $\varphi$. If $D_{A}(p)>K$, it is optimal to choose $q_{A}=D_{A}(p)-K$ (i.e. firm $A$ produces exactly the additional amount of $\eta$ that it needs to meet its demand) and transform $D_{A}(p)$ units of $\eta$ to $\varphi$. Therefore, $q_{A}=\max \left\{0, D_{A}(p)-K\right\}$.

Payoffs of firms in $\Gamma^{S}(\omega)$ : We can write the payoffs of firms by using the optimal values of $q_{A}, q_{B}$. Firm $B$ 's payoff has three components: (i) revenue from market $\varphi$, (ii) cost of producing $q_{B}$ units of $\eta$ to fulfill its demand and (iii) profit from supplying $K$ units of $\eta$ to $A$ at price $\omega$. Since firm $B$ 's unit cost of $\eta$ is $\underline{c}$ and $q_{B}=D_{B}(p)$, its payoff is

$$
\Pi_{B}^{\omega}(K, p)=p_{B} D_{B}(p)-\underline{c} q_{B}+(\omega-\underline{c}) K=\left(p_{B}-\underline{c}\right) D_{B}(p)+(\omega-\underline{c}) K
$$

Firm A's payoff also has three components: (i) revenue from market $\varphi$, (ii) cost of producing $q_{A}$ units of $\eta$ in-house to fulfill its demand and (iii) its payment to firm $B$ for acquiring $\eta$ at price $\omega$. Since firm $A$ 's unit cost of $\eta$ is $\bar{c}$ and $q_{A}=\max \left\{0, D_{A}(p)-K\right\}$, its payoff is

$$
\Pi_{A}^{\omega}(K, p)=p_{A} D_{A}(p)-\bar{c} q_{A}-\omega K=\left\{\begin{array}{l}
p_{A} D_{A}(p)-\omega K \text { if } D_{A}(p) \leq K \\
p_{A} D_{A}(p)-\bar{c}\left(D_{A}(p)-K\right)-\omega K \text { if } D_{A}(p)>K
\end{array}\right.
$$

We determine SPNE of $\Gamma^{S}(\omega)$ by backward induction. So we begin from stage II.

\subsubsection{Stage II of $\Gamma^{S}(\omega)$ : The Hotelling duopoly game $\mathbb{H}^{K}(\bar{c}, \underline{c})$}

The game $\mathbb{H}^{K}(\bar{c}, \underline{c})$ can be viewed as a Hotelling duopoly game in which firm $A$ has built a "capacity" of $K$ prior to the game. ${ }^{7}$ Specifically, in this game:

(i) Firm $B$ has constant unit cost $\underline{c}$.

(ii) Firm $A$ has capacity $K$ which is commonly known between $A$ and $B$. If the demand received by $A$ does not exceed $K$, it can fulfill the demand at zero unit cost. However, if its demand exceeds $K, A$ has to incur the cost $\bar{c}$ for every additional unit.

Observe from (13) that for firm $A, \omega K$ is the cost of capacity $K$ that it pays upfront to firm $B$ before stage II, so $\omega K$ plays no role stage II onwards. Similarly, from $(12),(\omega-\underline{c}) K$ is the profit that firm $B$ obtains upfront before stage II and it plays no role thereafter. Ignoring these terms, from (12), firm $B$ 's profit in $\mathbb{H}^{K}(\bar{c}, \underline{c})$ is

$$
\Phi_{B}^{K}(p)=\left(p_{B}-\underline{c}\right) D_{B}(p)
$$

\footnotetext{
${ }^{7}$ When $K=0, \mathbb{H}^{K}(\bar{c}, \underline{c})$ becomes the standard Hotelling duopoly game $\mathbb{H}(\bar{c}, \underline{c})$.
} 
From (13), firm $A^{\prime}$ 's profit in $\mathbb{H}^{K}(\bar{c}, \underline{c})$ is

$$
\Phi_{A}^{K}(p)=\left\{\begin{array}{l}
p_{A} D_{A}(p) \text { if } D_{A}(p) \leq K \\
p_{A} D_{A}(p)-\bar{c}\left(D_{A}(p)-K\right)=\left(p_{A}-\bar{c}\right) D_{A}(p)+\bar{c} K \text { if } D_{A}(p)>K
\end{array}\right.
$$

Therefore, firm $A$ has unit cost zero if $D_{A}(p) \leq K$, while its effective unit cost is $\bar{c}>0$ if $D_{A}(p)>K$.

Lemma 2 characterizes SPNE of the Hotelling duopoly game $\mathbb{H}^{K}(\bar{c}, \underline{c})$. Recall that the monopoly price, demand and profit at $\operatorname{cost} c$ are denoted by $\rho_{M}(c), \delta_{M}(c)$ and $\psi_{M}(c)$. Also recall that for $i \in\{A, B\}, p_{i}\left(c_{A}, c_{B}\right), D_{i}\left(c_{A}, c_{B}\right)$ and $\Phi_{i}\left(c_{A}, c_{B}\right)$ denote the SPNE price, market share and profit of firm $i$ in the standard Hotelling game $\mathbb{H}\left(c_{A}, c_{B}\right)$.

Lemma $2 \mathbb{H}^{K}(\bar{c}, \underline{c})$ has a unique SPNE that has the following properties.

(I) Under $U A$, firms $A$ and $B$ become two local monopolists. Firm B sets price $\rho_{M}(\underline{c})$, receives demand $\delta_{M}(\underline{c})$ and obtains profit $\psi_{M}(\underline{c})$.

(i) If $K<\delta_{M}(\bar{c})$, then firm $A$ sets price $\rho_{M}(\bar{c})$, receives demand $\delta_{M}(\bar{c})$ and obtains profit $\psi_{M}(\bar{c})+\bar{c} K$. Firm A fully utilizes its capacity $K$ and in addition produces $\delta_{M}(\bar{c})-K$ units of $\eta$ in-house to fulfill its demand.

(ii) If $K>\delta_{M}(0)$, then firm $A$ sets price $\rho_{M}(0)$, receives demand $\delta_{M}(0)$ and obtains profit $\psi_{M}(0)$. Firm $A$ does not utilize $K-\delta_{M}(0)$ units of its capacity and does not produce $\eta$ in-house.

(iii) If $K \in\left[\delta_{M}(\bar{c}), \delta_{M}(0)\right]$ then firm A sets price $\rho^{*}:=V-V_{0}-\tau K \in\left[\rho_{M}(0), \rho_{M}(\bar{c})\right]$, receives demand exactly equal to its capacity $K$ and obtains profit $\rho^{*} K$.

(II) Under $C A$

(i) If $K<D_{A}(\bar{c}, \underline{c})$, the prices and market shares of firms are the same as in the SPNE of $\mathbb{H}(\bar{c}, \underline{c})$. The profits are $\Phi_{A}^{K}=\Phi_{A}(\bar{c}, \underline{c})+\bar{c} K$ and $\Phi_{B}^{K}=\Phi_{B}(\bar{c}, \underline{c})$. Firm A fully utilizes its capacity $K$ and in addition produces $D_{A}(\bar{c}, \underline{c})-K$ units of $\eta$ in-house to fulfill its demand.

(ii) If $K>D_{A}(0, \underline{c})$, the prices, market shares and profits of firms are the same as in the SPNE of $\mathbb{H}(0, \underline{c})$. Firm $A$ does not utilize $K-D_{A}(0, \underline{c})$ units of its capacity and does not produce $\eta$ in-house.

(iii) If $K \in\left[D_{A}(\bar{c}, \underline{c}), D_{A}(0, \underline{c})\right]$, the prices of firms $A, B$ are

$$
p_{A}^{K}=3 \tau+\underline{c}-4 \tau K \text { and } p_{B}^{K}=2 \tau+\underline{c}-2 \tau K
$$

The market share of firm $A$ is $K$ and that of firm $B$ is $1-K$. Firm A fully utilizes its capacity $K$ and does not produce $\eta$ in-house. The profits are $\Phi_{A}^{K}=p_{A}^{K} K$ and $\Phi_{B}^{K}=$ $\left(p_{B}^{K}-\underline{c}\right)(1-K)$. 
Proof See the Appendix.

Observe that in the game $\mathbb{H}^{K}(\bar{c}, \underline{c})$, firm $B$ 's unit cost is always $\bar{c}$. For firm $A$, the minimum possible unit cost is 0 while the maximum possible unit cost is $\bar{c}$. Therefore, in an SPNE of $\mathbb{H}^{K}(\bar{c}, \underline{c})$ the maximum market share that firm $A$ can have is $D_{A}(0, \underline{c})$ (its SPNE market share in the standard Hotelling game $\mathbb{H}(0, \underline{c})$ ), while the minimum market share that it can have is $D_{A}(\bar{c}, \underline{c})$ (the corresponding market share in $\mathbb{H}(\bar{c}, \underline{c})$ ).

Lemma 2 shows that if the capacity of firm $A$ is too small $\left[K<D_{A}(\bar{c}, \underline{c})\right]$, building such a capacity gives $A$ no strategic advantage in $\mathbb{H}^{K}(\bar{c}, \underline{c})$ and the game yields the same SPNE outcome as $\mathbb{H}(\bar{c}, \underline{c})$. On the other hand, if the capacity is too large $\left[K>D_{A}(0, \underline{c})\right]$, the game results in the same SPNE outcome as $\mathbb{H}(0, \underline{c})$ where part of the capacity remains unutilized (so it is clear that building such large capacity cannot be optimal for firm $A$ ). Intermediate capacities $\left[D_{A}(\bar{c}, \underline{c}) \leq K \leq D_{A}(0, \underline{c})\right]$ have a commitment value in that for these values of $K$, the SPNE prices are such that the market share of $A$ in $\mathbb{H}^{K}(\bar{c}, \underline{c})$ exactly equals $K$. As a result, the capacity is fully utilized and $A$ does not produce $\eta$ in-house. Under covered markets, building such intermediate capacities indirectly establishes firm $A$ as the Stackelberg leader.

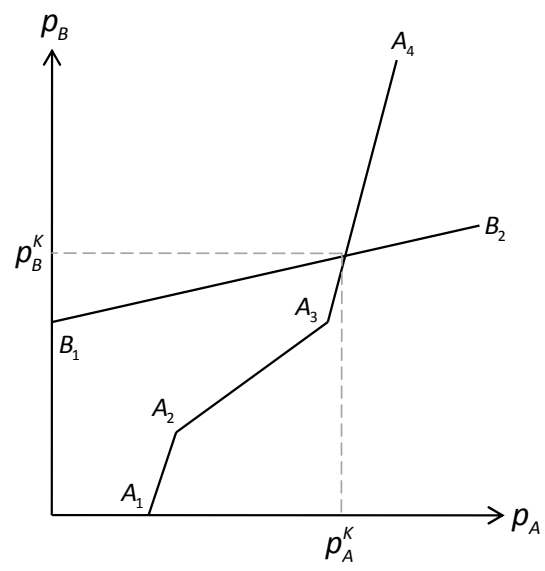

$1(a)$

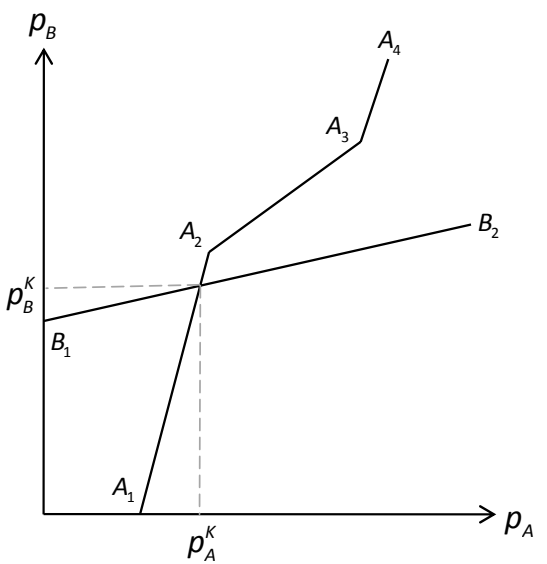

$1(b)$

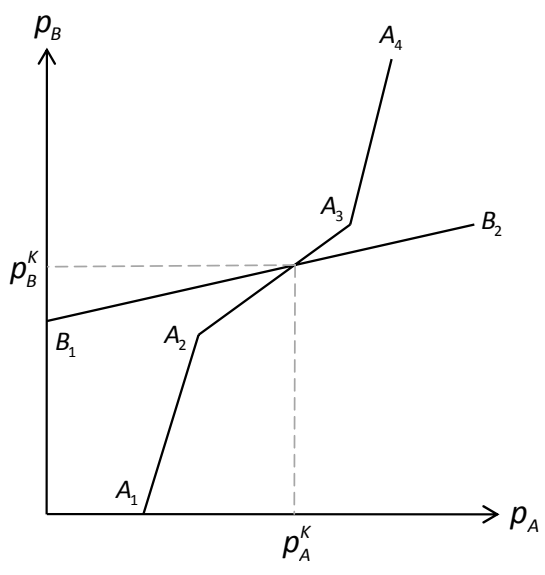

$1(c)$

$K<D_{A}(\bar{c}, \underline{c})$

$K>D_{A}(0, \underline{c})$

$K \in\left[D_{A}(\bar{c}, \underline{c}), D_{A}(0, \underline{c})\right]$

Figure 1: Hotelling Meets Stackelberg

Figure 1 above illustrates the Stackelberg effect. This figure identifies the $\operatorname{SPNE}\left(p_{A}^{K}, p_{B}^{K}\right)$ of $\mathbb{H}^{K}(\bar{c}, \underline{c})$ for different values of $K$. Since firm $B$ 's unit cost is fixed at $\underline{c}$, its best response is the same as in a standard Hotelling game, given by the line $B_{1} B_{2}$. Firm $A$ 's unit cost depends on its demand and its best response is a piecewise linear function that has three 
segments. If $B$ 's price $p_{B}$ is relatively small, $A$ 's best response is the same as in the standard Hotelling game $\mathbb{H}(0, \underline{c})$, given by $A_{1} A_{2}$. On the other hand, if $p_{B}$ is relatively large, $A$ 's best response is the same as in $\mathbb{H}(\bar{c}, \underline{c})$, given by $A_{3} A_{4}$. For intermediate values of $p_{B}$, its best response, $A_{2} A_{3}$, is such that the demand it receives is exactly equal to its capacity $K$.

Figure 1(a) corresponds to the case where $K<D_{A}(\underline{c}, \bar{c})$. For this case, $B_{1} B_{2}$ intersects the best response of $A$ at the segment $A_{3} A_{4}$. The SPNE is the same as in $\mathbb{H}(\bar{c}, \underline{c})$. Figure $1(\mathrm{~b})$ corresponds to the case where $K>D_{A}(0, \bar{c})$ and $B_{1} B_{2}$ intersects the best response of $A$ at the segment $A_{1} A_{2}$. The SPNE is the same as in $\mathbb{H}(0, \underline{c})$. Figure $1(\mathrm{c})$ corresponds to the case where $K \in\left[D_{A}(\underline{c}, \bar{c}), D_{A}(0, \bar{c})\right]$. For this case $B_{1} B_{2}$ intersects the best response of $A$ at the intermediate segment $A_{2} A_{3}$. This is where Hotelling meets Stackelberg. The SPNE $\left(p_{A}^{K}, p_{B}^{K}\right)$ is such that firm $A$ 's market share exactly equals its capacity $K$.

\subsubsection{Stage I of $\Gamma^{S}(\omega)$}

Now we move back to stage I of $\Gamma^{S}(\omega)$ where firm $A$ chooses its outsourcing order $K \in[0,1]$ of $\eta$. For any such $K$, in stage II, the game $\mathbb{H}^{K}(\bar{c}, \underline{c})$ is played whose unique SPNE is given in Lemma 2. Let $\Phi_{A}^{K}$ be the SPNE profit of firm $A$ in $\mathbb{H}^{K}(\bar{c}, \underline{c})$. In any SPNE play of $\Gamma^{S}(\omega)$, when firm $A$ orders $K$ units of $\eta$ from firm $B$ in stage I, its payoff is $\Phi_{A}^{K}-\omega K$ (its SPNE profit in $\mathbb{H}^{K}(\bar{c}, \underline{c})$ net of its payment $\omega K$ that it makes to firm $\left.B\right)$. By Lemma 2, under UA, this payoff is

$$
\Pi_{A}^{\omega}(K)= \begin{cases}\psi_{M}(\bar{c})+\bar{c} K-\omega K & \text { if } K<\delta_{M}(\bar{c}) \\ \rho^{*} K-\omega K & \text { if } K \in\left[\delta_{M}(\bar{c}), \delta_{M}(0)\right] \\ \psi_{M}(0)-\omega K & \text { if } K>\delta_{M}(0)\end{cases}
$$

Under CA, the payoff of $A$ is

$$
\Pi_{A}^{\omega}(K)= \begin{cases}\Phi_{A}(\bar{c}, \underline{c})+\bar{c} K-\omega K & \text { if } K<D_{A}(\bar{c}, \underline{c}) \\ p_{A}^{K} K-\omega K & \text { if } K \in\left[D_{A}(\bar{c}, \underline{c}), D_{A}(0, \underline{c})\right] \\ \Phi_{A}(0, \underline{c})-\omega K & \text { if } K>D_{A}(0, \underline{c})\end{cases}
$$

The payoff of firm $B$ is its SPNE profit in $\mathbb{H}^{K}(\bar{c}, \underline{c})$ plus its input supplier profit $(\omega-\underline{c}) K$. By Lemma 2, under UA, this payoff is

$$
\Pi_{B}^{\omega}(K)=\psi_{M}(\underline{c})+(\omega-\underline{c}) K
$$

Under CA, the payoff of $B$ is

$$
\Pi_{B}^{\omega}(K)= \begin{cases}\Phi_{B}(\bar{c}, \underline{c})+(\omega-\underline{c}) K & \text { if } K<D_{A}(\bar{c}) \\ \left(p_{B}^{K}-\underline{c}\right)(1-K)+(\omega-\underline{c}) K & \text { if } K \in\left[D_{A}(\bar{c}), D_{A}(0)\right] \\ \Phi_{B}(0, \underline{c})+(\omega-\underline{c}) K & \text { if } K>D_{A}(0)\end{cases}
$$

To determine SPNE of $\Gamma^{S}(\omega)$, in stage I, we solve the single-person decision problem of firm $A$ which is to choose $K \in[0,1]$ to maximize $\Pi_{A}^{\omega}(K)$ given by (17) under UA and (18) under CA.

Recall from Proposition 1 that when there are no contracts between firms, the standard Hotelling game $\mathbb{H}(\bar{c}, \underline{c})$ is played. The price, demand and payoff of firm $i \in\{A, B\}$ under no contracts are $p_{i}^{0}=p_{i}(\underline{c}, \bar{c}), D_{i}^{0}=D_{i}(\underline{c}, \bar{c})$ and $\Phi_{i}^{0}=\Phi_{i}(\bar{c}, \underline{c})$. 
Proposition 2 For any $\omega \in(\underline{c}, \bar{c}), \Gamma^{S}(\omega)$ has a unique SPNE. Let $K(\omega)$ be the amount of $\eta$ that firm $A$ orders from firm $B$ and for $i \in\{A, B\}$, let $p_{i}^{S}(\omega), D_{i}^{S}(\omega)$ and $\Pi_{i}^{S}(\omega)$ be the price, market share and payoff of firm $i$ in the SPNE. The SPNE has the following general properties.

(I) The demand that firm $A$ receives in the market $\varphi$ exactly equals the quantity of $\eta$ that it orders from firm B. Consequently firm A fully utilizes the amount of $\eta$ that it orders from $B$ and does not produce $\eta$ in-house.

(II) $\Pi_{A}^{S}(\omega)$ is decreasing and $\Pi_{B}^{S}(\omega)$ is increasing in $\omega$. Moreover $\Pi_{A}^{S}(\omega)>\Phi_{A}^{0}$, i.e., compared to the situation of no contracts, firm $A$ is better off.

(III) Compared to the situation of no contracts, no consumer is worse off.

The SPNE has the following specific properties.

(I) Suppose UA holds. Then for any $\omega \in(\underline{c}, \bar{c})$, firms $A$ and $B$ stay local monopolists, with $A$ having the effective unit cost $\omega$ and $B$ having cost $\underline{c}$. Consequently

$$
\begin{gathered}
p_{A}^{S}(\omega)=\rho_{M}(\omega), p_{B}^{S}(\omega)=\rho_{M}(\underline{c}), D_{A}^{S}(\omega)=\delta_{M}(\omega), D_{B}^{S}(\omega)=\delta_{M}(\underline{c}), \\
\Pi_{A}^{S}(\omega)=\psi_{M}(\omega) \text { and } \Pi_{B}^{S}(\omega)=\psi_{M}(\underline{c})+(\omega-\underline{c}) \delta_{M}(\omega)
\end{gathered}
$$

Consumers at location $x \in\left[0, \delta_{M}(\omega)\right)$ buy from $A, x \in\left[\delta_{M}(\omega), 1-\delta_{M}(\underline{c})\right]$ do not buy and $x \in\left(1-\delta_{M}(\underline{c}), 1\right]$ buy from $B$. Compared to the situation of no contracts, both firms are better off. Consumers at location $x \in\left[0, \delta_{M}(\omega)\right)$ (who buy from $A$ ) are better off and consumers at $x \in\left[\delta_{M}(\omega), 1\right]$ (who do not buy from $A$ ) are neither better off nor worse off.

(II) Suppose CA holds and let $\bar{c}-\underline{c}>(3 / 4) \tau$. There is $\widehat{c} \in(\underline{c}, \bar{c})$ such that

(a) If $\omega \in(\underline{c}, \widehat{c})$, then $K(\omega)=3 / 8-(\omega-\underline{c}) / 8 \tau \in\left(D_{A}(\bar{c}, \underline{c}), D_{A}(0, \underline{c})\right)$. The market share of firm $A$ is $K(\omega)$ and that of firm $B$ is $1-K(\omega)$. The prices are

$$
p_{A}^{S}(\omega)=(3 \tau+\underline{c}+\omega) / 2<p_{A}^{0} \text { and } p_{B}^{S}(\omega)=(5 \tau+3 \underline{c}+\omega) / 4<p_{B}^{0}
$$

Consequently all consumers are better off compared to the situation of no contracts. The payoffs are

$\Pi_{A}^{S}(\omega)=p_{A}^{S}(\omega) K(\omega)-\omega K(\omega)$ and $\Pi_{B}^{S}(\omega)=\left(p_{A}^{S}(\omega)-\underline{c}\right)(1-K(\omega))+(\omega-\underline{c}) K(\omega)$

Compared to the situation of no contracts, firm $A$ is better off. There is $\widetilde{c} \in(\underline{c}, \widehat{c})$ such that $\Pi_{B}^{S}(\omega) \gtreqless \Phi_{B}^{0} \Leftrightarrow \omega \gtreqless \widetilde{c}$, i.e., firm $B$ is better off only if $\omega \in(\widetilde{c}, \widehat{c})$.

(b) If $\omega \in[\widehat{c}, \bar{c})$, then $K(\omega)=D_{A}(\bar{c}, \underline{c})=D_{A}^{0}$. The prices and market shares of firms are exactly the same as in the case of no contracts. The payoffs are

$$
\Pi_{A}^{S}(\omega)=\Phi_{A}^{0}+(\bar{c}-\omega) D_{A}^{0} \text { and } \Pi_{B}^{S}(\omega)=\Phi_{B}^{0}+(\omega-\underline{c}) D_{A}^{0}
$$

Compared to the situation of no contracts, both firms are better off and consumers are neither better off nor worse off. 
(c) An outsourcing contract is strictly Pareto improving (both firms and all consumers are better off) if $\omega \in(\widetilde{c}, \widehat{c})$ and weakly Pareto improving (both firms are better off and no consumer is worse off) if $\omega \in[\widehat{c}, \bar{c})$.

(III) Suppose $C A$ holds and let $\bar{c}-\underline{c} \leq(3 / 4) \tau$. Then for any $\omega \in(\underline{c}, \bar{c}), K(\omega)=D_{A}(\bar{c}, \underline{c})$ and the conclusion is the same as in (II)(b). Consequently an outsourcing contract is weakly Pareto improving for all $\omega \in(\underline{c}, \bar{c})$.

Proof See the Appendix.

Firm $A$ 's outsourcing order of $\eta$ is equivalent to a capacity built by $A$ prior to price competition. The volume of the order transmits the information to $B$ that $A$ commits to maintain a specific share in the market $\varphi$. This commitment is credible, because following the order, the equilibrium prices are such that $A$ 's market share exactly equals its capacity. Consequently, the outsourcing order indirectly establishes firm $A$ as the Stackelberg leader in the market $\varphi$.

For firm $A$, the unit price of building the capacity is $\omega$. When $\omega$ is relatively large, the Stackelberg leader market share coincides with $A$ 's market share under no contracts. Since $\omega<\bar{c}$, it is optimal for $A$ to order its required $\eta$ from $B$. However, $A$ does not utilize its Stackelberg leader advantage since capacity building is relatively costly. For small values of $\omega$, the Stackelberg leader market share is larger than $A$ 's market share under no contracts. Here capacity building is less expensive which enables firm $A$ to utilize its leadership advantage. The Stackelberg leader market share is sustained in equilibrium by a lower price of firm $A$. Given that $A$ 's commitment to maintain this share is credible, $B$ 's equilibrium price is also set lower enabling it to stay competitive. As a result, prices of both firms fall. For firm $B$, this results in lower profit in the market $\varphi$ compared to the situation of no contracts. Therefore, $B$ would accept such a situation only if it can recover its losses in the final good market from its supplier profit, which is the case when $B$ is sufficiently efficient compared to $A$. Consequently, if the cost difference of two firms is sufficiently large (i.e., $\bar{c}-\underline{c}>(3 / 4) \tau)$, there are strictly Pareto improving outsourcing contracts.

\subsection{Technology transfer contract between $A$ and $B$}

When firm $B$ transfers its superior technology to firm $A$, both $A$ and $B$ can produce the intermediate good $\eta$ at lower cost $\underline{c}$. As in the case of outsourcing contract, we consider linear unit pricing contracts. The unit pricing contract for technology transfer is the unit royalty contract where the rate of royalty is denoted by $r$. The strategic interaction between $A$ and $B$ under technology transfer is described as follows.

Negotiation stage: In the beginning, firms $A$ and $B$ negotiate on the unit royalty $r$. Under the unit royalty contract, firm $A$ uses the superior technology of firm $B$. In return, $A$ pays $B$ the royalty $r$ for each unit of $\eta$ that it produces using the superior technology. So, firm $A$ 's effective unit cost of $\eta$ is $\underline{c}+r$. As $A$ can produce $\eta$ itself at unit cost $\bar{c}$, a royalty contract can lower its cost of production only if $\underline{c}+r<\bar{c}$ or $r<\bar{c}-\underline{c}$. On the other hand, firm $B$ can obtain a positive revenue from technology transfer only if $r>0$. So we restrict $r \in(0, \bar{c}-\underline{c})$. 
To compare royalty contracts with outsourcing contracts, it will be convenient to denote $\omega \equiv \underline{c}+r$. Then $\omega$ represents the effective unit cost of $\eta$ for firm $A$ under the superior technology, while $r=\omega-\underline{c}$ represents the unit profit of firm $B$ from technology transfer. Since $r \in(0, \bar{c}-\underline{c})$, we have $\omega \in(\underline{c}, \bar{c})$.

If firms do not agree on a price, firm $A$ produces the required $\eta$ entirely by itself at cost $\bar{c}$ and the game $\mathbb{H}(\bar{c}, \underline{c})$ is played in the final good market. If firms agree on a price $\omega \in(\underline{c}, \bar{c})$, firm $B$ transfers its superior technology to firm $A$ and the game $\Gamma^{T}(\omega)$ is played firms between $A$ and $B$.

Remark Observe that the interpretation of $\omega$ is the same as in outsourcing contracts. For firm $A, \omega$ is the unit cost of obtaining $\eta$ from firm $B$. For $B,(\omega-\underline{c})$ is the unit profit from supplying $\eta$ to $A$. The difference between outsourcing and technology transfer is that under outsourcing, $A$ chooses the quantity of $\eta$ and places its order with $B$ before firms set their prices for the final good market $\varphi$. Firm $B$ produces $\eta$ using its superior technology and supplies $\eta$ to $A$ at price $\omega$. In contrast, under technology transfer, $A$ uses the superior technology to produce $\eta$ itself after prices are set and its demand is known. This difference, which is generally overlooked in the existing literature, alters the strategic interaction and affects the prices of the final good $\varphi$.

The game $\Gamma^{T}(\omega)$ : It is an extensive form game that has the following stages.

Stage I: Firms $A$ and $B$ simultaneously announce prices $p_{A}, p_{B}$ for the final good market $\varphi$. For any $p \equiv\left(p_{A}, p_{B}\right)$, the demand received by firm $i \in\{A, B\}$ is $D_{i}(p)$, given by (3) under CA and (6) under UA.

Stage II: Observing $D_{i}(p)$, firms $A$ and $B$ simultaneously choose $q_{A}, q_{B} \geq 0$ where

$$
q_{i}=\text { the quantity of } \eta \text { that firm } i \text { produces in order to fulfill its demand of } \varphi
$$

The demand fulfilling constraints for firms $A, B$ are

$$
q_{A} \geq D_{A}(p) \text { and } q_{B} \geq D_{B}(p)
$$

Each firm fulfills its demand, profits are realized, firm $A$ makes its royalty payments to firm $B$ and the game terminates.

If firm $A$ produces $\eta$ using its pre-contract inferior technology, its unit cost is $\bar{c}$. If it produces $\eta$ using the superior technology, its unit cost is $\omega<\bar{c}$. So it is optimal for firm $A$ to produce $\eta$ entirely using the superior technology. Firm $B$ 's unit cost of producing $\eta$ is $\underline{c}>0$. Since both $\omega$ and $\bar{c}$ are positive, by (21), optimality requires that for $i \in\{A, B\}$, firm $i$ produces $q_{i}=D_{i}(p)$ units of $\eta$ and transforms $D_{i}(p)$ units to good $\varphi$ to fulfill its demand.

Payoffs of firms in $\Gamma^{T}(\omega)$ : Using optimal values of $q_{A}, q_{B}$, we can write the payoff of each firm. Firm $A$ 's payoff has two components: (i) revenue from market $\varphi$, (ii) total effective cost of producing $q_{A}$ units of $\eta$ to fulfill its demand. Observe that this total effective cost is $(\underline{c}+r) q_{A}=\omega q_{A}$, so it includes firm $A$ 's royalty payments to firm $B$. Therefore, the payoff of firm $A$ is

$$
\Pi_{A}^{\omega}(p)=p_{A} D_{A}(p)-\omega q_{A}=\left(p_{A}-\omega\right) D_{A}(p)
$$


Firm $B$ 's payoff has three components: (i) revenue from market $\varphi$, (ii) cost of producing $q_{B}$ units of $\eta$ to fulfill its demand and (iii) profit from technology transfer $r q_{A}=(\omega-\underline{c}) q_{A}$. Since firm $B$ 's unit cost of $\eta$ is $\underline{c}$ and $q_{i}=D_{i}(p)$, its payoff is

$$
\Pi_{B}^{\omega}(p)=p_{B} D_{B}(p)-\underline{c} q_{B}+(\omega-\underline{c}) q_{A}=\left(p_{B}-\underline{c}\right) D_{B}(p)+(\omega-\underline{c}) D_{A}(p)
$$

Covered Market: From (3), under CA, $D_{A}(p)+D_{B}(p)=1$. Using this fact in (23), the payoff of firm $B$ is

$$
\Pi_{B}^{\omega}(p)=\left(p_{B}-\underline{c}\right) D_{B}(p)+(\omega-\underline{c})\left(1-D_{B}(p)\right)=\left(p_{B}-\omega\right) D_{B}(p)+(\omega-\underline{c})
$$

Since $(\omega-\underline{c})$ is a constant, from (24) it follows that under CA, firm $B$ in effect solves the problem of a firm that has unit cost $\omega$. By (22), firm $A$ has unit cost $\omega$. Therefore, under CA, firms $A$ and $B$ effectively play the Hotelling duopoly game $\mathbb{H}(\omega, \omega)$ and SPNE of $\Gamma^{T}(\omega)$ coincides with SPNE of $\mathbb{H}(\omega, \omega)$ with the only modification that firm $B$ 's payoff has an additional constant $(\omega-\underline{c})$.

Remark Compared to situation of no contracts, firm $B$ 's effective unit cost increases from $\underline{c}$ to $\omega$. Unlike the case of outsourcing, the payments for technology transfer are not received upfront. To obtain relatively large royalty payments from technology transfer, $B$ has to ensure that $A$ 's market share in the market $\varphi$ is not too small. As the market share of $B$ necessarily falls with a larger share of $A$, it creates a distortion that raises the effective cost of $B$.

Uncovered Market: Under UA, firms $A$ and $B$ are two local monopolists. The demand received by $i \in\{A, B\}$ at price $p_{i}$ is $D_{M}^{p_{i}}$, given by (6). Then by (22), firm $A$ 's payoff is the monopoly profit under unit cost $\omega$, given by

$$
\Pi_{A}^{\omega}(p)=\left(p_{A}-\omega\right) D_{M}^{p_{A}}
$$

So it is optimal for $A$ to set the monopoly price $\rho_{M}(\omega)$. Then it receives the monopoly demand $\delta_{M}(\omega)$ and obtains the monopoly profit $\psi_{M}(\omega)$. From (23), firm $B$ 's payoff is

$$
\Pi_{B}^{\omega}(p)=\left(p_{B}-\underline{c}\right) D_{M}^{p_{B}}+(\omega-\underline{c}) D_{M}^{p_{A}}
$$

Observe that the term $(\omega-\underline{c}) D_{M}^{p_{A}}$ above (B's royalty revenue) does not depend on $p_{B}$. When $A$ optimizes, its demand is $\delta_{M}(\omega)$, so $B$ 's revenue from royalty equals $(\omega-\underline{c}) \delta_{M}(\omega)$. The first term, $\left(p_{B}-\underline{c}\right) D_{M}^{p_{B}}$, of (26) is $B$ 's profit as a local monopolist in the market $\varphi$. Therefore, it is optimal for $B$ to set the monopoly price $\rho_{M}(\underline{c})$. Then it receives demand $\delta_{M}(\underline{c})$ and obtains the payoff $\psi_{M}(\underline{c})+(\omega-\underline{c}) \delta_{M}(\omega)$ (monopoly profit plus revenue from royalty). Under uncovered market, firm $A$ 's demand does not depend on the price of firm $B$. Accordingly, the two components of firm $B$ 's payoff (profit in the market $\varphi$ and royalty revenue) are completely independent and firm $B$ 's unit cost stays at $\underline{c}$. Consequently, technology transfer does not have a distortive effect under uncovered market.

Proposition 3 For any $\omega \in(\underline{c}, \bar{c}), \Gamma^{T}(\omega)$ has a unique SPNE. For $i \in\{A, B\}$, let $p_{i}^{T}(\omega)$, $D_{i}^{T}(\omega)$ and $\Pi_{i}^{T}(\omega)$ be the price, market share and payoff of firm $i$ in the SPNE $\Gamma^{T}(\omega)$. The SPNE has the following properties.

(I) $\Pi_{A}^{T}(\omega)>\Phi_{A}^{0}$, i.e., compared to the situation of no contracts, firm $A$ is better off. 
(II) Suppose UA holds. Then for any $\omega \in(\underline{c}, \bar{c})$, firms $A$ and $B$ stay local monopolists, with $A$ having the effective unit cost $\omega$ and $B$ having the cost $\underline{c}$. The prices, market shares and payoffs are the same as in the SPNE of $\Gamma^{S}(\omega)$.

(III) Suppose CA holds. The prices and market shares of firms are the same as in the SPNE of $\mathbb{H}(\omega, \omega)$. Each firm sets the same price $\tau+\omega$ and obtains the same market share $1 / 2$. The payoffs are $\Pi_{A}^{T}(\omega)=\tau / 2$ and $\Pi_{B}^{T}(\omega)=\tau / 2+(\omega-\underline{c})$. Compared to the situation of no contracts, firm $A$ is better off. There are constants $\underline{c}<\underline{\theta}<\widehat{\theta}<\bar{\theta}<\bar{c}$ such that

(a) All consumers are better off if $\omega \in(\underline{c}, \underline{\theta})$ and all consumers are worse off if $\omega \in$ $(\bar{\theta}, \bar{c})$. If $\omega \in[\underline{\theta}, \bar{\theta}]$, then $\exists \lambda(\omega) \in(0,1 / 2]$ such that consumers at location $x \in[0, \lambda]$ are better off and $x \in(\lambda, 1]$ are worse off.

(b) $\Pi_{B}^{T}(\omega) \gtreqless \Phi_{B}^{0} \Leftrightarrow \omega \gtreqless \widehat{\theta}$, i.e., firm $B$ is better off only if $\omega \in(\widehat{\theta}, \bar{c})$.

(c) Whenever both firms prefer technology transfer over no contracts [i.e., if $\omega \in(\widehat{\theta}, \bar{c})]$, there are always some consumers who prefer no contracts over technology transfer. Consequently, there is no technology transfer contract that is Pareto improving (i.e., making both firms as well as all consumers better off).

Proof See the Appendix.

When the market is uncovered, firms $A$ and $B$ are two local monopolists and there is no strategic interaction in the market $\varphi$. Due to this reason, outsourcing and technology transfer result in the same outcome.

In the case of covered markets, firm $B$ 's profit from the market $\varphi$ depends on its own demand while its royalty revenue depends on the demand of firm $A$. Since the market size is fixed at 1 , a larger market share of $B$ necessarily leads to a smaller market share of $A$. Accordingly, there are two conflicting factors which affect $B$ 's payoff. An increase in $B$ 's own market share raises its profit in the market $\varphi$, however, it lowers the royalty revenue that it receives from $A$. For this reason, $B$ has an incentive to ensure that $A$ 's market share is not too small. This has a distortive effect which causes $B$ 's unit cost to rise to $\omega>\underline{c}$. As $A$ 's unit cost falls to $\omega<\bar{c}, A$ has an efficiency gain while $B$ has an efficiency loss. The resulting effect on consumers depends on which one of these opposing factors dominates. When $\omega$ is sufficiently small $(\omega<\underline{\theta})$, the efficiency gain of $A$ dominates, prices of both firms fall and all consumers are better off. When $\omega$ is sufficiently large $(\omega>\bar{\theta})$, the efficiency loss of $B$ dominates, prices of both firms rise and all consumers are worse off. For intermediate values of $\omega(\omega \in[\underline{\theta}, \bar{\theta}])$, the effect on consumers is ambiguous and it depends on their location. Consumers who are close to $A(x<\lambda)$ benefit from the efficiency gain of $A$ and therefore are better off. In contrast, consumers who are close to $B(x \geq \lambda)$ are adversely effected by the efficiency loss of $B$ and are worse off.

Observe that all consumers are better off under technology transfer compared to no contract only if $\omega$ is sufficiently small $(\omega<\underline{\theta})$. However, when $\omega$ is small, $B$ obtains a lower revenue from royalty. For this reason, firm $B$ prefers technology transfer over no contract only if $\omega$ is relatively large, in which case there are always some consumers who are worse off. This explains why there is no Pareto improving technology transfer contract. 


\subsection{Outsourcing versus technology transfer}

We have already shown in Proposition 3 that outsourcing and technology transfer lead to the same outcome under uncovered markets, making both firms and all consumers indifferent between the two. The next proposition compares these two contracts under covered markets.

Proposition 4 Suppose $C A$ holds. Let $\omega \in(\underline{c}, \bar{c})$. There are constants $\alpha, \beta, \underline{\theta}, \bar{\theta} \in(\underline{c}, \bar{c})$, satisfying $\alpha<\underline{\theta}<\beta<\bar{\theta}$ such that the following hold.

(I) If $\omega \in(\underline{c}, \alpha)$, both firms prefer outsourcing and if $\omega \in(\beta, \underline{c})$, both firms prefer technology transfer. If $\omega \in[\alpha, \beta]$, then firm A prefers technology transfer while firm $B$ prefers outsourcing.

(II) If $\omega \in(\underline{c}, \underline{\theta})$, all consumers prefer technology transfer and if $\omega \in(\bar{\theta}, \bar{c})$, all consumers prefer outsourcing. If $\omega \in[\underline{\theta}, \bar{\theta}]$, then $\exists \lambda(\omega) \in(0,1 / 2]$ such that consumers at location $x \in[0, \lambda]$ prefer technology transfer while consumers at $x \in(\lambda, 1]$ prefer outsourcing.

(III) Whenever both firms prefer a specific contract, all consumers prefer the other contract. Specifically if $\omega \in(\underline{c}, \alpha)$, both firms prefer outsourcing and all consumers prefer technology transfer. If $\omega \in(\beta, \bar{c})$, both firms prefer technology transfer and all consumers prefer outsourcing.

Proof See the Appendix.

Observe that under both outsourcing and technology transfer, $\omega$ is firm A's effective unit cost of obtaining $\eta$ and $(\omega-\underline{c})$ is firm $B$ 's unit profit from the market $\eta$. Comparing the contracts by keeping $\omega$ fixed implies that the effects of cost efficiency (for firm $A$ ) and supplier profits (for firm $B$ ) are the same across contracts.

Firm $A$ 's payoff under technology transfer is a constant $\tau / 2$, while its payoff under outsourcing is decreasing in $\omega$. Accordingly, $A$ prefers outsourcing for relatively small values of $\omega$ and technology transfer for relatively large values of $\omega$. Firm $B$ 's payoff has two components: profit from the final good market $\varphi$ and profit from the intermediate good market $\eta$. We have seen that under technology transfer, there is a distortive effect that raises the effective cost of $B$. As a result, the profit of $B$ in $\varphi$ is lower under technology transfer than outsourcing. Therefore, if $B$ is solely interested in the profits from the market $\varphi$, it would prefer outsourcing. On the other hand, if $B$ is only interested in the profits from the market $\eta$, it would prefer technology transfer. This is because the profit of $B$ from $\eta$ increases with the market share of $A$, which is higher under technology transfer. Consequently the two components of $B$ 's payoff conflict with each other. This trade-off is settled by the magnitude of $\omega$. When $\omega$ is relatively small, the profit from $\eta$ does not contribute significantly to $B$ 's payoff. As a result, the effect of the market $\varphi$ dominates and $B$ prefers outsourcing. On the other hand, when $\omega$ is relatively large, the profit from $\eta$ contributes significantly to $B$ 's payoff, so $B$ prefers technology transfer.

The preference of consumers between these two contracts depends on $\omega$. For relatively large values of $\omega$, prices under outsourcing are the same as in the case of no contracts, but prices under technology transfer exceed the no contract levels. Accordingly, all consumers prefer outsourcing for relatively large values of $\omega$. On the other hand, for relatively small values of $\omega$, prices under outsourcing may fall, but prices under technology transfer fall 
significantly below the no contract levels. Consequently all consumers prefer technology transfer for relatively small values of $\omega$. For intermediate values of $\omega$, the price of firm $A$ falls while the price of $B$ rises under technology transfer. As a result, the preference of consumers depends on their location as in Proposition 3. Finally it is shown that interest of consumers and incentives of firms conflict each other. Whenever both firms prefer one of the two contracts, all consumers prefer the other one.

\section{Concluding remarks}

This paper has compared two contracts that are frequently observed in industry practices: outsourcing and technology transfer. Departing from the existing literature, we have shown that these two contracts generate different strategic interactions that alter incentives of firms and have important effects on prices. Identifying the Stackelberg leadership effect in a Hotelling duopoly model, we have shown that there are always Pareto improving outsourcing contracts that make both firms and all consumers better off. In contrast, there are no Pareto improving technology transfer contracts. Due to the difference in strategic interaction between these contracts, the interest of consumers and incentives of firms move in completely opposite directions. When firms prefer outsourcing, all consumers prefer technology transfer and when firms prefer technology transfer, all consumers prefer outsourcing.

\section{Appendix}

We begin with Lemma A1 which will be used to prove Lemma 1.

Lemma A1 Suppose $C A$ holds and let $c_{A}, c_{B}<\tau$. Let $i, j \in\{A, B\}$ and $i \neq j$. In the game $\mathbb{H}\left(c_{A}, c_{B}\right)$ :

(i) The best response of firm $i$ to firm $j$ 's price $p_{j}$ is

$$
b_{c_{i}}\left(p_{j}\right)= \begin{cases}\left(p_{j}+\tau+c_{i}\right) / 2 & \text { if } p_{j} \leq 3 \tau+c_{i} \\ p_{j}-\tau & \text { if } p_{j}>3 \tau+c_{i}\end{cases}
$$

(ii) If $\left(p_{A}, p_{B}\right)$ is an SPNE of $\mathbb{H}\left(c_{A}, c_{B}\right)$, then $p_{i} \leq 3 \tau+c_{j}$ for $i, j \in\{A, B\}, i \neq j$.

Proof (i) By (3), if $p_{i} \leq p_{j}-\tau$, then $D_{i}=1$ and $i$ 's payoff is $p_{i}-c_{i}$, which is strictly increasing in $p_{i}$. If $p_{i} \geq p_{j}+\tau$, then $D_{i}=0$ and $i$ 's payoff is zero. Therefore, to determine best response of $i$, it is sufficient to consider $p_{i} \in\left[p_{j}-\tau, p_{j}+\tau\right]$. In that case, by (2) and (3), $D_{i}=\left(p_{j}-p_{i}+\tau\right) / 2 \tau$ and $i$ 's payoff is $\Phi_{i}=\left(p_{i}-c_{i}\right)\left(p_{j}-p_{i}+\tau\right) / 2 \tau$. Since $c_{i}<\tau$, we have $p_{j}+\tau>c_{i}$. Hence the unconstrained maximum of $\Phi_{i}$ with respect to $p_{i}$ is attained at $b\left(p_{j}\right)=\left(p_{j}+\tau+c_{i}\right) / 2<p_{j}+\tau$. The result in (27) follows by noting that $b\left(p_{j}\right) \geq p_{j}-\tau$ iff $p_{j} \leq 3 \tau+c_{j}$.

(ii) Suppose $\left(p_{A}, p_{B}\right)$ is an SPNE and $p_{j}>3 \tau+c_{i}$. Then by $(27), p_{i}=b_{c_{i}}\left(p_{j}\right)=p_{j}-\tau$. In that case, $D_{j}=0$ and firm $j$ obtains zero payoff. Let $j$ deviate to set the price $p_{j}^{\prime}=p_{i}=p_{j}-\tau$. Following this deviation, by (2) and (3), firm $j$ will receive demand $1 / 2$ and payoff $\left(p_{j}-c_{j}\right) / 2$. Since $p_{j}>3 \tau+c_{j}>c_{j}$, firm $j$ 's post-deviation payoff is positive. This shows that firm $j$ has improved its payoff following the deviation, a contradiction. 
Proof of Lemma 1(II) Suppose CA holds. By Lemma A1(ii), to find SPNE of $\mathbb{H}\left(c_{A}, c_{B}\right)$, it is sufficient to consider $p_{i} \leq 3 \tau+c_{i}$ for $i \in\{A, B\}$. Then by (27), the best response of $A$ is to set $p_{A}=\left(p_{B}+\tau+c_{A}\right) / 2$ and the best response of $B$ is to set $p_{B}=\left(p_{A}+\tau+c_{B}\right) / 2$. The system of best response equations has a unique solution where $p_{A}=\tau+\left(2 c_{A}+c_{B}\right) / 3$ and $p_{B}=\tau+\left(c_{A}+2 c_{B}\right) / 3$. This proves (i). Parts (ii)-(iv) follow directly from (i).

Lemma A2 will be used to prove Lemma 2.

Lemma A2 Suppose CA holds. Denote $p \equiv\left(p_{A}, p_{B}\right), g\left(p_{B}\right):=p_{B}+\tau-2 \tau K, \underline{p}(K):=$ $4 \tau K-\tau$ and $\bar{p}(K):=4 \tau K-\tau+\bar{c}$. In the game $\mathbb{H}^{K}(\underline{c}, \bar{c})$ :

(i) $D_{A}(p) \lesseqgtr K \Leftrightarrow p_{A} \gtreqless g\left(p_{B}\right)$.

(ii) The profit of firm $A$ is

$$
\Phi_{A}^{K}(p)= \begin{cases}p_{A}-\bar{c}+\bar{c} K & \text { if } p_{A}<p_{B}-\tau \\ \left(p_{A}-\bar{c}\right)\left(p_{B}-p_{A}+\tau\right) / 2 \tau+\bar{c} K & \text { if } p_{B}-\tau \leq p_{A}<g\left(p_{B}\right) \\ p_{A}\left(p_{B}-p_{A}+\tau\right) / 2 \tau & \text { if } g\left(p_{B}\right) \leq p_{A} \leq p_{B}+\tau \\ 0 & \text { if } p_{A}>p_{B}+\tau\end{cases}
$$

(iii) The best response of $A$ to $B$ 's price $p_{B}$ is

$$
b_{A}^{K}\left(p_{B}\right)= \begin{cases}b_{0}\left(p_{B}\right)=\left(p_{B}+\tau\right) / 2 & \text { if } p_{B}<\underline{p} \\ g\left(p_{B}\right) & \text { if } \leq p_{B} \leq \bar{p} \\ b_{\bar{c}}\left(p_{B}\right)=\left(p_{B}+\tau+\bar{c}\right) / 2 & \text { if } \overline{\bar{p}}<p_{B} \leq 3 \tau+\bar{c} \\ b_{\bar{c}}\left(p_{B}\right)=p_{B}-\tau & \text { if } p_{B}>3 \tau+\bar{c}\end{cases}
$$

(iv) Consider the demand that firm $A$ receives when it sets price $p_{A}=b_{A}^{K}\left(p_{B}\right)$. This demand is less than $K$ if $p_{B}<\underline{p}$, more than $K$ if $p_{B}>\bar{p}$ and exactly equals $K$ if $\underline{p} \leq p_{B} \leq \bar{p}$.

(v) The profit of firm $B$ is

$$
\Phi_{B}^{K}(p)= \begin{cases}p_{A}-\underline{c} & \text { if } p_{B}<p_{A}-\tau \\ \left(p_{B}-\underline{c}\right)\left(p_{A}-p_{B}+\tau\right) / 2 \tau & \text { if } p_{A}-\tau \leq p_{B} \leq p_{A}+\tau \\ 0 & \text { if } p_{B}>p_{A}+\tau\end{cases}
$$

(vi) The best response of $B$ to $A$ 's price $p_{A}$ is

$$
b_{B}^{K}\left(p_{A}\right)=b_{\underline{c}}\left(p_{A}\right)= \begin{cases}\left(p_{A}+\tau+\underline{c}\right) / 2 & \text { if } p_{A} \leq 3 \tau+\underline{c} \\ p_{A}-\tau & \text { if } p_{B}>3 \tau+\underline{c}\end{cases}
$$

(vii) If $\left(p_{A}, p_{B}\right)$ is an $S P N E$ of $\mathbb{H}\left(c_{A}, c_{B}\right)$, then $p_{A} \leq 3 \tau+\underline{c}$ and $p_{B} \leq 3 \tau+\bar{c}$.

Proof (i) Observe that since $K \in[0,1]$, we have $p_{B}-\tau \leq g\left(p_{B}\right) \leq p_{B}+\tau$. It follows from (3) that if $p_{A} \leq p_{B}-\tau$, then $D_{A}(p)=1 \geq K$ and if $p_{A} \geq p_{B}+\tau$, then $D_{A}(p)=0 \leq K$. Now consider $p_{A} \in\left[p_{B}-\tau, p_{B}+\tau\right]$. Then from (2) and (3), we have $D_{A}(p)=\left(p_{B}-p_{A}+\tau\right) / 2 \tau \lesseqgtr$ $K \Leftrightarrow p_{A} \gtreqless g\left(p_{B}\right)$. This completes the proof of (i).

(ii) Observe from (15) that $\Phi_{A}^{K}(p)=\left(p_{A}-\bar{c}\right) D_{A}(p)+\bar{c} K$ if $D_{A}(p) \geq K$. The first expression of $(28)$ follows by noting that $D_{A}(p)=1 \geq K$ for $p_{A}<p_{B}-\tau$. Since $D_{A}(p)=$ $\left(p_{B}-p_{A}+\tau\right) / 2 \tau \geq K$ for $p_{A} \in\left[p_{B}-\tau, g\left(p_{B}\right)\right]$ (by part (i)), the second expression follows. 
Again from (15), $\Phi_{A}^{K}(p)=p_{A} D_{A}^{p}$ if $D_{A}(p) \leq K$. Since $D_{A}(p)=\left(p_{B}-p_{A}+\tau\right) / 2 \tau \leq K$ for $p_{A} \in\left[g\left(p_{B}\right), p_{B}+\tau\right]$ (by part (i)), the third expression of (28) follows. The last expression follows by noting that $D_{A}(p)=0 \leq K$ for $p_{A}>p_{B}+\tau$.

(iii) It follows from (28) that $\Phi_{A}^{K}(p)$ is strictly increasing for $p_{A} \leq p_{B}-\tau$ and it equals zero for $p_{A} \geq p_{B}+\tau$. Therefore, to determine best response of $A$, it is sufficient to consider $p_{A} \in\left[p_{B}-\tau, p_{B}+\tau\right]$.

Let $E_{1}=\left[p_{B}-\tau, g\left(p_{B}\right)\right]$ and $E_{2}=\left[g\left(p_{B}\right), p_{B}+\tau\right]$. Observe from (28) that for $p_{A} \in E_{1}$, firm $A$ 's effective unit cost is $\bar{c}$ and its problem is the same as in the standard Hotelling game $\mathbb{H}\left(\bar{c}, c_{B}\right)$. Taking $i=A$ and $c_{A}=\bar{c}$ in (27) of Lemma A1, the unconstrained maximum of $\Phi_{A}^{K}(p)$ over $p_{A} \in E_{1}$ is attained at $p_{A}=b_{\bar{c}}\left(p_{B}\right)$. Note from (27) that if $p_{B}>3 \tau+\bar{c}$, then $b_{\bar{c}}\left(p_{B}\right)=p_{B}-\tau$. If $p_{B} \leq 3 \tau+\bar{c}$, then $b_{\bar{c}}\left(p_{B}\right)=\left(p_{B}+\tau+\bar{c}\right) / 2 \lesseqgtr g\left(p_{B}\right) \Leftrightarrow p_{B} \gtreqless \bar{p}$ where $\bar{p}:=4 \tau K-\tau+\bar{c} \leq 3 \tau+\bar{c}$. Hence we conclude that

$$
\arg \max _{p_{A} \in E_{1}} \Phi_{A}^{K}(p)= \begin{cases}g\left(p_{B}\right) & \text { if } p_{B}<\bar{p} \\ \left(p_{B}+\tau+\bar{c}\right) / 2 & \text { if } \bar{p} \leq p_{B} \leq 3 \tau+\bar{c} \\ p_{B}-\tau & \text { if } p_{B}>3 \tau+\bar{c}\end{cases}
$$

Observe from (28) that for $p_{A} \in E_{2}$, firm $A$ 's effective unit cost is 0 and its problem is the same as in the standard Hotelling game $\mathbb{H}\left(0, c_{B}\right)$. Taking $i=A$ and $c_{A}=0$ in (27) of Lemma A1, the unconstrained maximum of $\Phi_{A}^{K}(p)$ over $p_{A} \in E_{2}$ is attained at $p_{A}=b_{0}\left(p_{B}\right)$. Note from (27) that if $p_{B}>3 \tau$, then $b_{0}\left(p_{B}\right)=p_{B}-\tau \leq g\left(p_{B}\right)$, so the maximum is attained at $p_{A}=g\left(p_{B}\right)$. If $p_{B} \leq 3 \tau$, then $b_{0}\left(p_{B}\right)=\left(p_{B}+\tau\right) / 2 \gtreqless g\left(p_{B}\right) \Leftrightarrow p_{B} \lesseqgtr \underline{p}$ where $\underline{p}:=4 \tau K-\tau \leq$ $3 \tau$. Hence we conclude that

$$
\arg \max _{p_{A} \in E_{2}} \Phi_{A}^{K}(p)= \begin{cases}\left(p_{B}+\tau\right) / 2 & \text { if } p_{B} \leq \underline{p} \\ g\left(p_{B}\right) & \text { if } p_{B}>\underline{p}\end{cases}
$$

As $g\left(p_{B}\right) \in E_{1} \cap E_{2}$, choosing $p_{A}=g\left(p_{B}\right)$ is feasible for both $E_{1}$ and $E_{2}$. Using this fact, the result in (29) follows from (32)-(33).

(iv) It follows from (iii) that $b_{A}^{K}\left(p_{B}\right)>g\left(p_{B}\right)$ if $p_{B}<\underline{p}, b_{A}^{K}\left(p_{B}\right)<g\left(p_{B}\right)$ if $p_{B}>\bar{p}$ and $b_{A}^{K}\left(p_{B}\right)=g\left(p_{B}\right)$ if $\underline{p} \leq p_{B} \leq \bar{p}$. Using this fact, the result follows from (i).

(v)-(vi) Noting that firm $B$ 's constant unit cost of $\eta$ is $\underline{c}$, (30) follows from (2) and (3), and (31) follows from (27) by taking $i=B$ and $c_{B}=\underline{c}$.

(vii) Follows from (29) and (31) by the same reasoning as the proof of Lemma A1(ii).

Proof of Lemma 2 (I) Suppose UA holds. Recall from (9) that when a monopolist has cost $c$, the monopoly price, demand and profit are $\rho_{M}(c)=\left(V-V_{0}+c\right) / 2, \delta_{M}(c)=\left(V-V_{0}-c\right) / 2 \tau$ and $\psi_{M}(c)=\left(V-V_{0}-c\right)^{2} / 4 \tau$. Noting that firm $B$ is a local monopolist with unit cost $\underline{c}$, the result for $B$ is immediate.

By (15), firm $A$ 's profit is as follows, where $D_{M}^{p_{A}}=\left(V-V_{0}-p_{A}\right) / \tau$ is the demand received by a monopolist at price $p_{A}$.

$$
\Phi\left(p_{A}\right)= \begin{cases}p_{A} D_{M}^{p_{A}} & \text { if } D_{M}^{p_{A}} \leq K \\ \left(p_{A}-\bar{c}\right) D_{M}^{p_{A}}+\bar{c} K & \text { if } D_{M}^{p_{A}}>K\end{cases}
$$

Observe that $D_{M}^{p_{A}} \gtreqless K \Leftrightarrow p_{A} \lesseqgtr \rho^{*}$ where $\rho^{*}:=V-V_{0}-\tau K$. If $p_{A} \leq \rho^{*}$, then $D_{M}^{p_{A}} \geq K$ and by (34), firm $A$ solves the problem of a monopolist that has cost $\bar{c}$. If $p_{A} \geq \rho^{*}$, then 
$D_{M}^{p_{A}} \leq K$ and by (34), firm $A$ solves the problem of a monopolist that has cost 0 . Hence we conclude that

$$
\arg \max _{p_{A} \leq \rho^{*}} \Phi\left(p_{A}\right)=\min \left\{\rho^{*}, \rho_{M}(\bar{c})\right\} \text { and } \arg \max _{p_{A} \geq \rho^{*}} \Phi\left(p_{A}\right)=\max \left\{\rho^{*}, \rho_{M}(0)\right\}
$$

Also observe that $\rho^{*} \gtreqless \rho_{M}(\bar{c}) \Leftrightarrow \delta_{M}(\bar{c}) \gtreqless K$ and $\rho^{*} \gtreqless \rho_{M}(0) \Leftrightarrow \delta_{M}(0) \gtreqless K$.

(i) Let $K<\delta_{M}(\bar{c})<\delta_{M}(0)$. Then $\rho^{*}>\rho_{M}(\bar{c})>\rho_{M}(0)$. By (35), it follows that $\arg \max _{p_{A} \leq \rho^{*}} \Phi\left(p_{A}\right)=\rho_{M}(\bar{c})$ and $\arg \max _{p_{A} \geq \rho^{*}} \Phi\left(p_{A}\right)=\rho^{*}$. Hence it is optimal for $A$ to set price $\rho_{M}(\bar{c})$. Then it receives demand $\delta_{M}(\bar{c})>K$, produces $\delta_{M}(\bar{c})-K$ units of $\eta$ in-house and by (34), obtains the profit $\psi_{M}(\bar{c})+\bar{c} K$.

(ii) Let $K>\delta_{M}(0)>\delta_{M}(\bar{c})$. Then $\rho^{*}<\rho_{M}(0)<\rho_{M}(\bar{c})$. By (35), it follows that $\arg \max _{p_{A} \leq \rho^{*}} \Phi\left(p_{A}\right)=\rho^{*}$ and $\arg \max _{p_{A} \geq \rho^{*}} \Phi\left(p_{A}\right)=\rho_{M}(0)$. Hence it is optimal for $A$ to set price $\rho_{M}(0)$. Then it receives demand $\delta_{M}(0)<K$, does not produce $\eta$ in-house and $K-\delta_{M}(0)$ units of $\eta$ are not utilized. By (34), $A$ obtains the profit $\psi_{M}(0)$.

(iii) Let $K \in\left[\delta_{M}(\bar{c}), \delta_{M}(0)\right]$. Then $\rho^{*} \in\left[\rho_{M}(0), \rho_{M}(\bar{c})\right]$ and by $(35), \arg \max _{p_{A} \leq \rho^{*}} \Phi\left(p_{A}\right)=$ $\arg \max _{p_{A} \geq \rho^{*}} \Phi\left(p_{A}\right)=\rho^{*}$. Hence it is optimal for $A$ to set price $\rho^{*}$. Then it receives demand exactly $K$ and by (34), obtains the profit $\rho^{*} K$.

(II) Suppose CA holds. Using Lemma A2(vii), to find SPNE of $\mathbb{H}^{K}(\underline{c}, \bar{c})$, consider $p_{A} \leq$ $3 \tau+\underline{c}$ and $p_{B} \leq 3 \tau+\bar{c}$. From $(29)$, firm $A$ 's best response $b_{A}^{K}\left(p_{B}\right)$ is piecewise linear with three segments: $b_{\bar{c}}\left(p_{B}\right)$ (if $\left.p_{B}>\bar{p}\right), b_{0}\left(p_{B}\right)$ (if $p_{B}<\underline{p}$ ) and $g\left(p_{B}\right)$ (if $p_{B} \in[p, \bar{p}]$ ). From (31), firm $B$ 's best response is linear, given by $b_{\underline{c}}\left(p_{B}\right)$. Hence any segment of $b_{A}^{K}\left(p_{B}\right)$ can intersect $b_{\underline{c}}\left(p_{B}\right)$ at most once. It will be useful to recall that for $i=1,2$, the SPNE price and market share of firm $i$ in $\mathbb{H}\left(c_{A}, c_{B}\right)$ are denoted by $p_{i}\left(c_{A}, c_{B}\right)$ and $D_{i}\left(c_{A}, c_{B}\right)$.

Note that $b_{\bar{c}}\left(p_{B}\right)$ is the best response of $A$ in the standard Hotelling game $\mathbb{H}(\bar{c}, \underline{c})$. Firm $B$ 's best response in this game is $b_{\underline{c}}\left(p_{A}\right)$. By Lemma $1(\mathrm{II})$, the unique solution of the system $\left(p_{A}=b_{\bar{c}}\left(p_{B}\right), p_{B}=b_{\underline{c}}\left(p_{A}\right)\right)$ has $p_{A}=p_{A}(\bar{c}, \underline{c})$ and $p_{B}=p_{B}(\bar{c}, \underline{c})$. We note that $p_{B}(\bar{c}, \underline{c}) \gtreqless \bar{p} \Leftrightarrow$ $K \lesseqgtr D_{A}(\bar{c}, \underline{c})$. Hence we have an SPNE with $p_{B}>\bar{p}$ iff $p_{B}=p_{B}(\bar{c}, \underline{c})>\bar{p}$, which holds iff $K<D_{A}(\bar{c}, \underline{c})$ (see Figure 1(a)). For this case, firm $A$ fully utilizes its capacity $K$ and moreover produces $D_{A}(\bar{c}, \underline{c})-K$ units of $\eta$ in-house to meet its demand.

Next observe that $b_{0}\left(p_{B}\right)$ is the best response of $A$ in the standard Hotelling game $\mathbb{H}(0, \underline{c})$. Firm $B$ 's best response in this game is $b_{\underline{c}}\left(p_{A}\right)$. By Lemma $1(\mathrm{II})$, the unique solution of the system $\left(p_{A}=b_{0}\left(p_{B}\right), p_{B}=b_{\underline{c}}\left(p_{A}\right)\right)$ has $p_{A}=p_{A}(0, \underline{c})$ and $p_{B}=p_{B}(0, \underline{c})$. We note that $p_{B}(0, \underline{c}) \lesseqgtr \underline{p} \Leftrightarrow K \gtreqless D_{A}(0, \underline{c})$. Hence we have an SPNE with $p_{B}<\underline{p}$ iff $p_{B}=p_{B}(0, \underline{c})<\underline{p}$, which holds iff $K>D_{A}(0, \underline{c})$ (see Figure 1(b)). For this case, firm $A$ does not utilize $K-D_{A}(0, \underline{c})$ units of its capacity and does not produce $\eta$ in-house.

Finally observe that the unique solution of $\left(p_{A}=g\left(p_{B}\right), p_{B}=b_{\underline{c}}\left(p_{A}\right)\right)$ has $p_{A}=3 \tau+\underline{c}-$ $4 \tau K$ and $p_{B}=2 \tau+\underline{c}-2 \tau K$. Note that $2 \tau+\underline{c}-2 \tau \gtreqless \underline{p} \Leftrightarrow K \lesseqgtr D_{A}(0, \underline{c})$ and $2 \tau+\underline{c}-2 \tau K \lesseqgtr$ $\bar{p} \Leftrightarrow K \gtreqless D_{A}(\bar{c}, \underline{c})$. Hence we have an SPNE with $p_{B} \in[\underline{p}, \bar{p}]$ iff $p_{B}=2 \tau+\underline{c}-2 \tau K \in[\underline{p}, \bar{p}]$, which holds iff $K \in\left[D_{A}(\bar{c}, \underline{c}), D_{A}(0, \underline{c})\right]$ (see Figure 1(c)). For this case, firm $A$ 's SPNE market share exactly equals its capacity $K$. It fully utilizes its capacity and does not produce $\eta$ in-house.

The results (II)[(i)-(iii)] of Lemma 2 follow from the conclusions of the last three paragraphs. 
Proof of Proposition 2 We prove the specific properties (I)-(III) of Proposition 2. The general properties will follow directly from the specific properties.

(I) Suppose UA holds. To determine SPNE of $\mathbb{H}^{K}(\underline{c}, \bar{c})$, we solve the problem of firm $A$ in stage 1 where $A$ chooses $K \in[0,1]$ to maximize $\Pi_{A}^{\omega}(K)$ given in (17). Observe that (i) since $\omega<\bar{c}, \Pi_{A}^{\omega}(K)$ is strictly increasing for $K \leq \delta_{M}(\bar{c})$ and (ii) since $\omega>\underline{c}>0$, it is strictly decreasing for $K \geq \delta_{M}(0)$. So it is sufficient to consider $K \in\left[\delta_{M}(\bar{c}), \delta_{M}(0)\right]$. Then by (17),

$$
\Pi_{A}^{\omega}(K)=\rho^{*} K-\omega K=\left(V-V_{0}-\omega-\tau K\right) K
$$

whose unique maximizer is attained at $K=\delta_{M}(\omega):=\left(V-V_{0}-\omega\right) / 2 \tau \in\left(\delta_{M}(\bar{c}), \delta_{M}(0)\right)$. Consequently the price set by $A$ in the market $\varphi$ is $\rho_{M}(\omega)$ and $A$ obtains $\psi_{M}(\omega)$. Since $B$ 's cost is $\underline{c}$, it sets price $\rho_{M}(\underline{c})$, receives demand $\delta_{M}(\underline{c})$ and obtains payoff $\psi_{M}(\underline{c})+(\omega-\underline{c}) \delta_{M}(\omega)$ (profit from $\varphi$ plus profit from $A$ 's order of $K=\delta_{M}(\omega)$ ). As $\omega<\bar{c}$, we have $\rho_{M}(\omega)<\rho_{M}(\bar{c})$. Hence consumers who buy from $A$ are better off. All other consumers are neither better off nor worse off.

(II) Suppose CA holds. Here we solve the problem of firm $A$ in Stage 1 where $A$ chooses $K \in[0,1]$ to maximize $\Pi_{A}^{\omega}(K)$ given in (18). As in the last case, $\Pi_{A}^{\omega}(K)$ is strictly increasing for $K \leq D_{A}(\bar{c}, \underline{c})$ and strictly decreasing for $K \geq D_{A}(0, \underline{c})$. So it is sufficient to consider $K \in\left[D_{A}(\bar{c}, \underline{c}), D_{A}(0, \underline{c})\right]$. Then by (16) and (18),

$$
\Pi_{A}^{\omega}(K)=p_{A}^{K} K-\omega K=(3 \tau+\underline{c}-\omega-4 \tau K) K .
$$

Since $\tau>\bar{c}>\omega$, we have $3 \tau+\underline{c}-\omega>0$. Hence the unconstrained maximum of $\Pi_{A}^{\omega}(K)$ is attained at $\Theta(\omega):=3 / 8-(\omega-\underline{c}) / 8 \tau<D_{A}(0, \underline{c})=1 / 2+\underline{c} / 6 \tau$. Therefore, over $K \in$ $\left[D_{A}(\bar{c}, \underline{c}), D_{A}(0, \underline{c})\right]$, the maximizer of $\Pi_{A}^{\omega}(K)$ is

$$
K(\omega)=\min \left\{\Theta(\omega), D_{A}(\bar{c}, \underline{c})\right\}
$$

Comparing $\Theta(\omega)$ with $D_{A}(\bar{c}, \underline{c})=1 / 2-(\bar{c}-\underline{c}) / 6 \tau$, we have

$$
\Theta(\omega) \gtreqless D_{A}(\bar{c}, \underline{c}) \Leftrightarrow \omega \lesseqgtr \widehat{c} \text { where } \widehat{c} \equiv 4 \bar{c} / 3-\underline{c} / 3-\tau
$$

Observe that (i) $\bar{c}-\widehat{c}=\tau+\underline{c} / 3-\bar{c} / 3>0$ and (ii) $\widehat{c}-\underline{c}=(4 / 3)[\bar{c}-\underline{c}-(3 / 4) \tau]$. Hence

$$
\widehat{c} \gtreqless \underline{c} \Leftrightarrow \bar{c}-\underline{c} \gtreqless(3 / 4) \tau
$$

From (36), (37) and (38), we conclude that

(1) If $\bar{c}-\underline{c} \leq(3 / 4) \tau$, then $K(\omega)=D_{A}(\bar{c}, \underline{c})$ for all $\omega \in(\underline{c}, \bar{c})$.

(2) If $\bar{c}-\underline{c}>(3 / 4) \tau$, then (i) $K(\omega)=\Theta(\omega)$ for $\omega \in(\underline{c}, \widehat{c})$ and (ii) $K(\omega)=D_{A}(\bar{c}, \underline{c})$ for $\omega \in[\widehat{c}, \bar{c})$.

The results of (II)(b) and (III) of Prop 2 follow from (1) and (2)(ii) above.

To prove (II)(a) of Prop 2, observe from (2)(i) above that if $\bar{c}-\underline{c}>(3 / 4) \tau$ and $\omega \in(\underline{c}, \widehat{c})$, then $K(\omega)=\Theta(\omega) \in\left(D_{A}(\bar{c}, \underline{c}), D_{A}(0, \underline{c})\right)$ where $\Theta(\omega)=3 / 8-(\omega-\underline{c}) / 8 \tau$. From Lemma 2(iii), it follows that the market share of firm $A$ is $\Theta(\omega)$, while the share of $B$ is $1-\Theta(\omega)$. Taking $K=\Theta(\omega)$ in (16), it follows that

$$
p_{A}^{S}(\omega)=(3 \tau+\underline{c}+\omega) / 2 \text { and } p_{B}^{S}(\omega)=(5 \tau+3 \underline{c}+\omega) / 4 .
$$


Note from Prop 1(II) that under no contracts, the prices are

$$
p_{A}^{0}=\tau+\bar{\theta} \text { and } p_{B}^{0}=\tau+\underline{\theta} \text { where } \underline{\theta} \equiv(2 \underline{c}+\bar{c}) / 3 \text { and } \bar{\theta} \equiv(\underline{c}+2 \bar{c}) / 3 .
$$

As $\omega<\widehat{c}$, we have $p_{A}^{0}-p_{A}^{S}(\omega)=(\widehat{c}-\omega) / 2>0$ and $p_{B}^{0}-p_{B}^{S}(\omega)=(\widehat{c}-\omega) / 4>0$. Hence all consumers are better off.

The payoffs of the firms are obtained from (18) and (20) by using the values of $p_{A}^{S}, p_{B}^{S}$ and taking $K=\Theta(\omega)$. Note from (18) that since $\omega<\bar{c}$, firm $A$ can be better off compared to the case of no contracts by simply choosing $K=D_{A}(\bar{c}, \underline{c})$. Therefore, under its optimal choice $K(\omega)$, it must be better off. The payoff of $B$ is

$$
\Pi_{B}^{S}(\omega)=(5 \tau+\omega-\underline{c})^{2} / 32 \tau+(\omega-\underline{c})(3 \tau+\underline{c}-\omega) / 8 \tau
$$

which is strictly increasing in $\omega$. Recall from Prop 1 that under no contracts, $B$ obtains $\Pi_{B}^{0}=(3 \tau+\bar{c}-\underline{c})^{2} / 18 \tau$. Hence $\Pi_{B}^{S}(\omega)-\Pi_{B}^{0}$ is strictly increasing in $\omega$. Note that since $\tau>\bar{c}$ and $\bar{c}-\underline{c}>(3 / 4) \tau$, we have

$$
\begin{gathered}
\Pi_{B}^{S}(\underline{c})-\Pi_{B}^{0}=(27 \tau-4 \bar{c}+4 \underline{c})[(3 / 4) \tau-\bar{c}+\underline{c}] / 72 \tau<0 \text { and } \\
\Pi_{B}^{S}(\widehat{c})-\Pi_{B}^{0}=2(3 \tau-\bar{c}+\underline{c})[\bar{c}-\underline{c}-(3 / 4) \tau] / 9 \tau>0 .
\end{gathered}
$$

Hence $\exists \widetilde{c} \in(0, \widehat{c})$ such that $\Pi_{B}^{S}(\omega) \gtreqless \Pi_{B}^{0} \Leftrightarrow \omega \gtreqless \widetilde{c}$. This completes the proof of II(a). Part (II)(c) follows from (II)(a) and (b).

Proof of Proposition 3 We prove parts (II) and (III). Part (I) follows from (II) and (III).

(II) Suppose UA holds. We have already proved in the main text before the statement of Prop 3 that under UA, it is optimal for $A$ to set price $\rho_{M}(\omega)$. Then it receives demand $\delta_{M}(\omega)$ and obtains payoff $\psi_{M}(\omega)$. It is optimal for $B$ to set price $\rho_{M}(\omega)$. Then it receives demand $\delta_{M}(\underline{c})$ and obtains payoff $\psi_{M}(\underline{c})+(\omega-\underline{c}) \delta_{M}(\omega)$. Noting that the prices and the payoffs are same as in the SPNE of $\Gamma^{S}(\omega)$, the proof of (II) is complete.

(III) Suppose CA holds. From (22) and (24) it follows that under CA, SPNE prices and market shares of $\Gamma^{T}(\omega)$ coincide with SPNE of game $\mathbb{H}(\omega, \omega)$. Taking $c_{A}=\omega$ and $c_{B}=\omega$ in Lemma 1, the prices and market shares are obtained. Using the SPNE prices and market shares, the payoffs are obtained from (22) and (24). Now we prove results (a)-(c).

(III)(a) Recall from Prop 1 that when there are no contracts, the SPNE prices under CA are $p_{A}^{0}=\tau+\bar{\theta}$ and $p_{B}^{0}=\tau+\underline{\theta}$ where $\underline{c}<\underline{\theta}<\bar{\theta}<\bar{c}$ with

$$
\underline{\theta} \equiv(2 \underline{c}+\bar{c}) / 3 \text { and } \bar{\theta} \equiv(\underline{c}+2 \bar{c}) / 3
$$

The SPNE prices in $\Gamma^{T}(\omega)$ are $p_{A}^{T}(\omega)=p_{B}^{T}(\omega)=\tau+\omega$. Therefore, if $\omega \in(\underline{c}, \underline{\theta})$, then $p_{i}^{T}(\omega)<p_{i}^{0}$ for $i \in\{A, B\}$ and all consumers prefer technology transfer over no contracts. If $\omega \in(\bar{\theta}, \bar{c}), p_{i}^{T}(\omega)>p_{i}^{0}$ for $i \in\{A, B\}$ and all consumers prefer no contracts over technology transfer.

If $\omega \in[\underline{\theta}, \bar{\theta}]$, then $p_{A}^{T}(\omega) \leq p_{A}^{0}$ and $p_{B}^{T}(\omega) \geq p_{B}^{0}$. Note that in $\Gamma^{T}(\omega)$, firm $A^{\prime}$ 's SPNE market share is $1 / 2>D_{A}^{0}$ where $D_{A}^{0}=D_{A}(\bar{c}, \underline{c})$ is the SPNE market share of firm $A$ under no contracts. Consider the consumers at $x \in\left[0, D_{A}^{0}\right]$. In both cases (i.e., no contracts and $\left.\Gamma^{T}(\omega)\right)$, they buy from firm $A$. As $p_{A}^{T}(\omega) \leq p_{A}^{0}$, these consumers are better off in $\Gamma^{T}(\omega)$. Next consider the consumers at $x \in[1 / 2,1]$. In both cases they buy from firm $B$. As $p_{B}^{T}(\omega) \geq p_{B}^{0}$, 
they are worse off in $\Gamma^{T}(\omega)$. Finally consider any consumer at $x \in\left[D_{A}^{0}, 1 / 2\right)$. When there is no contract, such a consumer buys from firm $B$ to obtain the net utility

$$
U_{x}^{0}=V-p_{B}^{0}-\tau(1-x)=V-(\tau+\underline{\theta})-\tau(1-x)
$$

In $\Gamma^{T}(\omega)$, this consumer buys from $A$ to obtain the net utility

$$
U_{x}^{T}=V-p_{A}^{S}-\tau x=V-(\tau+\omega)-\tau x
$$

Hence $U_{x}^{T}-U_{x}^{0} \gtreqless 0 \Leftrightarrow x \lesseqgtr \lambda(\omega):=1 / 2-(\omega-\underline{\theta}) / 2 \tau$. Since $\omega \geq \underline{\theta}$, we have $\lambda(\omega) \leq 1 / 2$. Since $D_{A}^{0}=1 / 2-(\bar{c}-\underline{c}) / 6 \tau$, from $(40)$ we have $\lambda(\omega)-D_{A}^{0}=(\bar{\theta}-\omega) / 2 \tau \geq 0$ (since $\omega \leq \bar{\theta}$ ). Thus $\lambda(\omega) \in\left[D_{A}^{0}, 1 / 2\right]$. We conclude that consumers at $x \in\left[D_{A}^{0}, \lambda\right]$ prefer technology transfer while consumers at $x \in(\lambda, 1 / 2)$ prefer no contracts. Since consumers at $x \in\left[0, D_{A}^{0}\right)$ prefer technology transfer and $x \in[1 / 2,1]$ prefer no contracts, the proof of (III)(a) is complete.

(III)(b) Note that $\Pi^{T}(\omega)=\tau / 2+(\omega-\underline{c})$ and $\Phi_{B}^{0}=(3 \tau+\bar{c}-\underline{c})^{2} / 18 \tau$. Denoting $f(\omega):=$ $\Pi^{T}(\omega)-\Phi_{B}^{0}$, note that $f(\omega)$ is increasing, $f(\underline{c})=-(\bar{c}-\underline{c})(6 \tau+\bar{c}-\underline{c}) / 18 \tau<0$ and $f(\bar{c})=$ $(\bar{c}-\underline{c})(12 \tau-\bar{c}+\underline{c}) / 18 \tau>0$. Hence $\exists \widehat{\theta} \in(\underline{c}, \bar{c})$ such that $\Pi^{T}(\omega) \gtreqless \Phi_{B}^{0} \Leftrightarrow \omega \gtreqless \widehat{\theta}$. Standard computations show that $\widehat{\theta} \equiv \underline{\theta}+(\bar{c}-\underline{c})^{2} / 18 \tau$. Therefore, $\widehat{\theta}>\underline{\theta}$. Comparing $\widehat{\hat{\theta}}$ with $\bar{\theta}$ from (40), we have $\bar{\theta}-\widehat{\theta}=(\bar{c}-\underline{c})(6 \tau+\underline{c}-\bar{c}) / 18 \tau>0$ proving that $\underline{\theta}<\widehat{\theta}<\bar{\theta}$.

(III)(c) Follows from parts (a) and (b).

Lemma A3 will be used to prove Proposition 4.

Lemma A3 Suppose CA holds. Let $\omega \in(\underline{c}, \bar{c})$. There are constants $\alpha, \beta \in(\underline{c}, \bar{c})$ such that

(I) $\Pi_{A}^{T}(\omega) \gtreqless \Pi_{A}^{S}(\omega) \Leftrightarrow \omega \gtreqless \alpha$.

(II) $\Pi_{B}^{T}(\omega) \gtreqless \Pi_{B}^{S}(\omega) \Leftrightarrow \omega \gtreqless \beta$.

Proof Denote $\sigma:=\bar{c}-\underline{c}$ and observe that $\sigma<\tau$. Also for $i \in\{A, B\}$ we denote $\Delta_{i}(\omega):=$ $\Pi_{i}^{T}(\omega)-\Pi_{i}^{S}(\omega)$. We prove the lemma by showing that there are constants $\alpha, \beta \in(\underline{c}, \bar{c})$ such that (I) $\Delta_{A}(\omega) \gtreqless 0 \Leftrightarrow \omega \gtreqless \alpha$ and (II) $\Delta_{B}(\omega) \gtreqless 0 \Leftrightarrow \omega \gtreqless \beta$.

(I) Recall that $\Pi_{A}^{T}(\omega)=\tau / 2$ for all $\omega \in\left(\underline{c}, \bar{c}\right.$ ) (Prop 3) and $\Pi_{A}^{S}(\omega)$ is strictly decreasing in $\omega$ (Prop 2). Hence $\Delta_{A}(\omega)$ is strictly increasing in $\omega$. To determine $\Pi_{A}^{S}(\omega)$, we consider the following possible cases where $\widehat{c} \equiv 4 \bar{c} / 3-\underline{c} / 3-\tau$.

Case $1 \sigma>(3 / 4) \tau$ :

Subcase $\mathbf{1}(\mathbf{a}) \omega \in(\underline{c}, \widehat{c})$ : For this case, from Prop 2, we have

$$
\Pi_{A}^{S}(\omega)=\left(p_{A}^{S}-\omega\right) K(\omega)=(3 \tau+\underline{c}-\omega)^{2} / 16 \tau, \text { so that } \Delta_{A}(\omega)=\tau / 2-(3 \tau+\underline{c}-\omega)^{2} / 16 \tau
$$

Observe that $\Delta_{A}(\underline{c})=-\tau / 16<0$ and $\Delta_{A}(\widehat{c})=\left[2 \sigma^{2}-(3 \tau-2 \sigma)^{2}\right] / 18 \tau$. Hence $\Delta_{A}(\widehat{c}) \gtreqless 0 \Leftrightarrow$ $\sigma \gtreqless 3(2-\sqrt{2}) \tau / 2$. We have the following two possibilities.

(i) If $3(2-\sqrt{2}) \tau / 2<\sigma<\tau$, then $\Delta_{A}(\widehat{c})>0$. Since $\Delta_{A}(\underline{c})<0, \exists \widehat{\alpha} \in(\underline{c}, \widehat{c})$ such that $\Delta_{A}(\omega) \gtreqless 0 \Leftrightarrow \omega \gtreqless \widehat{\alpha}$. Standard computations show that

$$
\widehat{\alpha} \equiv(3-2 \sqrt{2}) \tau+\underline{c}
$$


(ii) If $(3 / 4) \tau<\sigma \leq 3(2-\sqrt{2}) \tau / 2$, then $\Delta_{A}(\widehat{c}) \leq 0$. Hence $\Delta_{A}(\omega)<0$ for all $\omega \in(\underline{c}, \widehat{c})$.

Subcase 1(b) $\omega \in[\widehat{c}, \bar{c})$ : For this case $K(\omega)=D_{A}(\bar{c}, \underline{c})$ and $\Pi_{A}^{S}(\omega)=\Phi_{A}^{0}+(\bar{c}-\omega) D_{A}^{0}=$ $(3 \tau-\sigma)^{2} / 18 \tau+(\bar{c}-\omega)(3 \tau-\sigma) / 6 \tau$. Hence we have

$$
\Delta_{A}(\omega)=\tau / 2-(3 \tau-\sigma)^{2} / 18 \tau-(\bar{c}-\omega)(3 \tau-\sigma) / 6 \tau
$$

Note that $\Delta_{A}(\bar{c})=\sigma(6 \tau-\sigma) / 18 \tau>0$. Noting that $\Delta_{A}(\omega)$ is continuous, from the last case we know that $\Delta_{A}(\widehat{c}) \gtreqless 0 \Leftrightarrow \sigma \gtreqless 3(2-\sqrt{2}) \tau / 2$. Again we consider two possibilities.

(i) If $3(2-\sqrt{2}) \tau / 2<\sigma<\tau$, then $\Delta_{A}(\widehat{c})>0$. Hence $\Delta_{A}(\omega)>0$ for all $\omega \in[\widehat{c}, \bar{c})$.

(ii) If $(3 / 4) \tau<\sigma \leq 3(2-\sqrt{2}) \tau / 2$, then $\Delta_{A}(\widehat{c}) \leq 0$. Since $\Delta_{A}(\bar{c})>0, \exists \widetilde{\alpha} \in(\widehat{c}, \bar{c})$ such that $\Delta_{A}(\omega) \gtreqless 0 \Leftrightarrow \omega \gtreqless \widetilde{\alpha}$. Standard computations show that

$$
\widetilde{\alpha} \equiv 2 \underline{c} \tau /(3 \tau-\sigma)-\sigma(\underline{c}+\bar{c}) / 3(3 \tau-\sigma)+\bar{c} / 3
$$

Case $2 \sigma \leq(3 / 4) \tau$ : For this case, by Prop $2, \Pi_{A}^{S}(\omega)$ and $\Delta_{A}(\omega)$ are the same as in Subcase 1 (b) for all $\omega \in(\underline{c}, \bar{c})$. From Subcase $1(\mathrm{~b}), \Delta_{A}(\bar{c})>0$. Noting that $\Delta_{A}(\underline{c})=-\sigma(3 \tau-\sigma) / 18 \tau<$ 0 , we conclude that $\exists \widetilde{\alpha} \in(\underline{c}, \bar{c})$ [given in $(42)$ ] such that $\Delta_{A}(\omega) \gtreqless 0 \Leftrightarrow \omega \gtreqless \widetilde{\alpha}$.

Define

$$
\alpha:= \begin{cases}\widehat{\alpha} & \text { if } 3(2-\sqrt{2}) \tau / 2<\sigma<\tau \\ \widetilde{\alpha} & \text { if } \sigma \leq 3(2-\sqrt{2}) \tau / 2\end{cases}
$$

Using (43), for $3(2-\sqrt{2}) \tau / 2<\sigma<\tau$, the result follows from Subcases [1(a)(i)]-[1(b)(i)], for $(3 / 4) \tau<\sigma \leq 3(2-\sqrt{2}) \tau / 2$, it follows from Subcases [1(a)(ii)]-[1(b)(ii)] and for $\sigma \leq(3 / 4) \tau$, from Case 2.

(II) Recall from Prop 3 that $\Pi_{B}^{T}(\omega)=\tau / 2+(\omega-\underline{c})$ for all $\omega \in(\underline{c}, \bar{c})$. To determine $\Pi_{B}^{S}(\omega)$, we consider the following possible cases.

Case $1 \sigma>(3 / 4) \tau$ :

Subcase $\mathbf{1}(\mathbf{a}) \omega \in(\underline{c}, \widehat{c})$ : For this case, by Prop 2 and (39), we have

$$
\begin{gathered}
\Pi_{B}^{S}(\omega)=(5 \tau-\underline{c}+\omega)^{2} / 32 \tau+(\omega-\underline{c})[3 / 8-(\omega-\underline{c}) / 8 \tau] \text { and } \\
\Delta_{B}(\omega)=\tau / 2+(\omega-\underline{c})[5 / 8+(\omega-\underline{c}) / 8 \tau]-(5 \tau-\underline{c}+\omega)^{2} / 32 \tau
\end{gathered}
$$

Note that $\Delta_{B}(\omega)$ is increasing in $\omega$. Now observe that $\Delta_{B}(\widehat{c})=\left[(\tau+2 \sigma)^{2}-13 \tau^{2}\right] / 24 \tau<$ $\left(9 \tau^{2}-13 \tau^{2}\right) / 24 \tau<0$ (since $\left.\sigma<\tau\right)$. Hence $\Delta_{B}(\omega)<0$ for all $\omega \in(\underline{c}, \widehat{c})$.

Subcase 1(b) $\omega \in[\widehat{c}, \underline{c})$ : For this case, by Prop 2, we have $\Pi_{B}^{S}(\omega)=\Phi_{B}^{0}+(\omega-\underline{c}) D_{B}^{0}=$ $(3 \tau+\sigma)^{2} / 18 \tau+(\omega-\underline{c})(3 \tau+\sigma) / 6 \tau$, so that

$$
\Delta_{B}(\omega)=\tau / 2+(\omega-\underline{c})(1 / 2+\sigma / 6 \tau)-(3 \tau+\sigma)^{2} / 18 \tau
$$

Note that $\Delta_{B}(\omega)$ is increasing in $\omega$. We know from the last case that $\Delta_{B}(\widehat{c})<0$. Observing that $\Delta_{B}(\bar{c})=\sigma(3 \tau+2 \sigma) / 18 \tau>0$, we conclude that $\exists \beta \in(\widehat{c}, \bar{c})$ such that $\Delta_{B}(\omega) \gtreqless 0 \Leftrightarrow$ $\omega \gtreqless \beta$. Standard computations show that

$$
\beta \equiv 2 \bar{c} \tau /(3 \tau+\sigma)+\sigma(\underline{c}+\bar{c}) / 3(3 \tau+\sigma)+\underline{c} / 3
$$


Case $2 \sigma \leq(3 / 4) \tau$ : For this case, by Prop 2, $\Pi_{B}^{S}(\omega)$ and $\Delta_{B}(\omega)$ are the same as in Subcase $1(\mathrm{~b})$ for all $\omega \in(\underline{c}, \bar{c})$. From Subcase $1(\mathrm{~b})$, we know that $\Delta_{B}(\bar{c})>0$. Noting that $\Delta_{B}(\underline{c})=-\sigma(6 \tau+\sigma) / 18 \tau<0$, we conclude that $\exists \beta \in(\underline{c}, \bar{c})$ [given in (44)] such that $\Delta_{B}(\omega) \gtreqless 0 \Leftrightarrow \omega \gtreqless \beta$.

The result for $\sigma>(3 / 4) \tau$ follows from Subcases $1(\mathrm{a})-(\mathrm{b})$ and for $\sigma \leq(3 / 4) \tau$, it follows from Case 2.

Proof of Proposition 4 (I) We prove (I) from Lemma A3 by showing that $\alpha<\beta$. Denote $\sigma:=\bar{c}-\underline{c}$. First let $\sigma>3(2-\sqrt{2}) \tau / 2>(3 / 4) \tau$. Then by Case 1 of the proof Lemma A3(II), $\beta>\widehat{c}$ and by subcases [1(a)(ii)]-[1(b)(ii)] and (43) of the proof of Lemma A3(I), $\alpha=\widehat{\alpha}<\widehat{c}$. Hence $\beta>\alpha$. Next consider $\sigma \leq 3(2-\sqrt{2}) \tau / 2$. Then by (43), $\alpha=\widetilde{\alpha}$. By (42) and (44) we have $\beta-\widetilde{\alpha}=\sigma\left(9 \tau^{2}+\sigma^{2}\right) / 3\left(9 \tau^{2}-\sigma^{2}\right)>0$.

(II) To prove (II), we consider the following possible cases.

Case $1 \sigma>(3 / 4) \tau$ :

Subcase 1(a) $\omega \in(\underline{c}, \widehat{c})$ : For this case, from Prop 2, the SPNE prices in $\Gamma^{S}(\omega)$ are

$$
p_{A}^{S}(\omega)=(3 \tau+\underline{c}+\omega) / 2 \text { and } p_{B}^{S}(\omega)=(5 \tau+3 \underline{c}+\omega) / 4
$$

From Prop 3, the SPNE prices in $\Gamma^{T}(\omega)$ are $p_{A}^{T}(\omega)=p_{B}^{T}(\omega)=\tau+\omega$. Hence $p_{A}^{S}(\omega)-p_{A}^{T}(\omega)=$ $(\tau+\underline{c}-\omega) / 2>0$ and $p_{B}^{S}(\omega)-p_{B}^{T}(\omega)=(\tau+3 \underline{c}-3 \omega) / 4 \gtreqless 0 \Leftrightarrow \omega \lesseqgtr \tau / 3+\underline{c}$. Note from (37) that $\widehat{c} \equiv 4 \bar{c} / 3-\underline{c} / 3-\tau$. Hence $(\tau / 3+\underline{c})-\widehat{c}=(4 / 3)(\tau+\underline{c}-\bar{c})>0$. Therefore, for all $\omega \in(\underline{c}, \widehat{c})$, we have $\omega<(\tau / 3+\underline{c})$ and hence that $p_{B}^{S}(\omega)>p_{B}^{T}(\omega)$. Consequently for this case all consumers prefer technology transfer over outsourcing.

Subcase 1(b) $\omega \in[\widehat{c}, \bar{c})$ : Note from Prop 2 that for this case the SPNE prices in $\Gamma^{S}(\omega)$ are the same as in the case of no contracts. Therefore, comparing outsourcing and technology transfer for consumers is the same as comparing technology transfer with no contracts and we can use the results of Prop 3 (III)(a).

Since $\underline{\theta}=(2 \underline{c}+\bar{c}) / 3$ and $\widehat{c}=4 \bar{c} / 3-\underline{c} / 3-\tau$, we have $\underline{\theta}-\widehat{c}=\tau+\underline{c}-\bar{c}>0$, i.e., $\underline{\theta}>\widehat{c}$. From Prop 2(III)(a), it then follows that (i) if $\omega \in[\widehat{c}, \underline{\theta})$, then all consumers prefer technology transfer over outsourcing, (ii) if $\omega \in(\bar{\theta}, \bar{c})$, then all consumers prefer outsourcing over technology transfer and (iii) if $\omega \in[\underline{\theta}, \bar{\theta}]$, there is $\lambda(\omega) \in(0,1 / 2]$ such that consumers at $x \in[0, \lambda]$ prefer technology transfer and $x \in(\lambda, 1]$ prefer outsourcing. Combining the conclusions of Subcases 1(a)-(b), the proof for the case of $\sigma>(3 / 4) \tau$ is complete.

Case $2 \sigma \leq(3 / 4) \tau$ : For this case, for all $\omega \in(\underline{c}, \bar{c})$, the SPNE prices in $\Gamma^{S}(\omega)$ are the same as in the case when there are no contracts. The result then follows directly from Prop $3(\mathrm{III})(\mathrm{a})$.

(III) The result will follow from (I)-(II) if we can show that $\alpha<\underline{\theta}<\beta<\bar{\theta}$. Note from (43) that if $\sigma>3(2-\sqrt{2}) \tau / 2>(3 / 4) \tau$, then $\alpha=\widehat{\alpha}<\widehat{c}$. Since $\underline{\theta}>\widehat{c}$, for this case, we have $\underline{\theta}>\alpha$. Next consider $\sigma \leq 3(2-\sqrt{2}) \tau / 2$. Then by (43), $\alpha=\widetilde{\alpha}$. By (42) we have $\underline{\theta}-\widetilde{\alpha}=\sigma^{2} / 3(3 \tau-\sigma)>0$. From (44) and (40), it follows that $\bar{\theta}-\beta=\sigma^{2} / 3(3 \tau+\sigma)>0$ and $\beta-\underline{\theta}=\tau \sigma /(3 \tau+\sigma)>0$. This completes the proof of Proposition 4. 


\section{References}

Anderson, S.P., de Palma, A. and Thisse, J-F. 1992. Discrete Choice Theory of Product Differentiation. The MIT Press, Cambridge, Massachusetts.

Arya, A., Mittendorf, B. and Sappington, D., 2007. Outsourcing, vertical integration, and price vs. quantity competition. International Journal of Industrial Organization, 26, 1-16.

Baake, P., Oechssler, J. and Schenk, C., 1999. Explaining cross-supplies. Journal of Economics, 70, 37-60.

Chen, Y., Dubey, P. and Sen, D. 2009. Outsourcing induced by strategic competition. MPRA Paper 14899, University Library of Munich, Germany.

Chen, Y., Ishikawa, J. and Yu, Z., 2004. Trade liberalization and strategic outsourcing. Journal of International Economics, 63, 419-436.

Domberger, S., 1998. The Contracting Organization: A Strategic Guide to Outsourcing. Oxford University Press.

Gabszewicz, J.J. and Thisse, J-F. 1992. Location. In: Aumann, R.J. \& Hart, S. (Eds). Handbook of Game Theory with Economic Applications, Vol. 1, North Holland, Amsterdam.

Jarillo, J.C., 1993. Strategic Networks: Creating Borderless Organization. ButterworthHeinmann.

Kreps, D.M. and Scheinkman, J.A. 1983. Quantity precommitment and Bertrand competition yield Cournot outcomes. Bell Journal of Economics, 14, 326-333.

Mendi, P. 2005. The structure of payments in technology transfer contracts: evidence from Spain. Journal of Economics and Management Strategy, 14, 403-429.

Nagaoka, S. 2005. Determinants of high-royalty contracts and the impact of stronger protection of intellectual property rights in Japan. Journal of the Japanese and International Economies, 19, 233-254.

Robinson, M., Kalakota, R. 2004. Offshore Outsourcing: Business Models, ROI and Best Practices, Mivar Press, Inc.

Shy, O. and Stenbacka, R., 2003. Strategic outsourcing. Journal of Economic Behavior and Organization, 50, 203-224.

Vagadia, B. 2007. Outsourcing to India-A Legal Handbook, Springer.

Vishwasrao, S. 2007. Royalties vs. fees: How do firms pay for foreign technology? International Journal of Industrial Organization, 25, 741-759. 